\title{
TWISTED $K$-THEORY
}

\author{
OLD AND NEW
}

by

\section{Max Karoubi}

\section{Some history and motivation about this paper}

The subject " $K$-theory with local coefficients", now called "twisted $K$-theory", was introduced by P. Donovan and the author in [DK] 37 years ago. It associates to a compact space $X$ and a "local coefficient system"

$$
\alpha \in \operatorname{GBr}(X)=\mathbf{Z} / 2 \times H^{1}(X ; \mathbf{Z} / 2) \times \operatorname{Tors}\left(H^{3}(X ; \mathbf{Z})\right)
$$

an abelian group $K^{\alpha}(X)$ which generalizes the usual Grothendieck-Atiyah-Hirzebruch $K$-theory of $X$ when we restrict $\alpha$ being in $\mathbf{Z} / 2$ (cf. [A1]). This "graded Brauer group" $G B r(X)$ has the following group structure : if $\alpha=\left(\varepsilon, w_{1}, W_{3}\right)$ and $\alpha^{\prime}=\left(\varepsilon^{\prime}, w_{1}^{\prime}, W_{3}^{\prime}\right)$ are two elements, one defines the sum $\alpha+\alpha^{\prime}$ as $\left(\varepsilon+\varepsilon^{\prime}, w_{1}+w_{1}^{\prime}, W_{3}+W_{3}^{\prime}+\beta\left(w_{1} w_{1}^{\prime}\right)\right)$, where $\beta: H^{2}(X ; \mathbf{Z} / 2)$ $\rightarrow H^{3}(X ; \mathbf{Z})$ is the Bockstein homomorphism. With this definition, one has a generalized cupproduct

$$
K^{\alpha}(X) \times K^{\alpha^{\prime}}(X) \rightarrow K^{\alpha+\alpha^{\prime}}(X)
$$

The motivation for this definition is to give in $K$-theory a satisfactory Thom isomorphism and Poincaré duality pairing which are analogous to the usual ones in cohomology with local coefficients. More precisely, as proved in [K1] (a precursor to twisted $K$-theory), if $V$ is a real vector bundle on a compact space $X$ with a positive metric, the $K$-theory of the Thom space of $V$ is isomorphic to a certain group $K^{C(V)}(X)$ associated to the Clifford bundle $C(V)$, viewed as a bundle of $\mathbf{Z} / 2$-graded algebras. A careful analysis of this group shows that it depends only on the class of $C(V)$ in $\operatorname{GBr}(X)$, the three invariants being respectively the rank of $V$ mod. 2, $w_{1}(V)$ and $\beta\left(w_{2}(V)\right)=W_{3}(V)$, where $w_{1}(V), w_{2}(V)$ are the first two Stiefel-Whitney classes of $V$. In

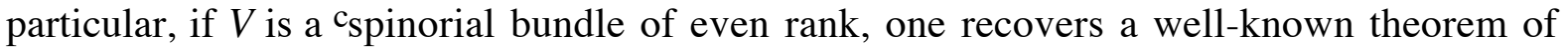
Atiyah and Hirzebruch [AH]. On the other hand, if $X$ is a compact connected manifold, it is well-known that such a Thom isomorphism theorem induces a pairing between K-groups

$$
K^{\alpha}(X) \times K^{\alpha}(X) \rightarrow \mathbf{Z}
$$

if $\alpha+\alpha^{\prime}$ is the class of $-C(V)$ in $\operatorname{GBr}(X)$, where $V$ is the tangent bundle of $X$.

The necessity to revisit these ideas comes from a new interest in the subject because of its relations with Physics [Wi], as shown by the number of recent publications. However, for these applications, the first definition recalled above is not complete since the coefficient system is restricted to the torsion elements of $H^{3}(X ; \mathbf{Z})$. As it was pointed out by J. Rosenberg [R] and later on by C. Laurent, J.-L. Tu, P. Xu [LTX], M. F. Atiyah and G. Segal [AS], this restriction is in fact not necessary. In order to avoid it, one may use for instance the Atiyah-Janich theorem $[\mathrm{A} 1][\mathrm{J}]$ about the representability of $K$-theory by the space of Fredholm operators (already mentioned in [DK], but not for this purpose). 
In the present paper, the road we choose is to give a synthesis between different viewpoints on the subject : [DK], [R], [CTX] and [AS] (partially of course) ${ }^{1}$. We hope we have been "pedagogical" in some sense to the non experts.

However, we dont pretend to be exhaustive. For instance, one should add to the previous references [BCMMS] for the relations with the theory of gerbes and D-branes in Physics; $\mathrm{V}$. Mathai and D. Stevenson [MS] who use the Chern character defined by A. Connes and M. Karoubi in order to prove an isomorphism between twisted $K$-theory and a computable "twisted cohomology" (at least rationally) ; D. Freed, M. Hopkins and C. Teleman [FHT] about the relation between loop groups and twisted K-theory and many other works which are mentioned inside the paper and in the list of references at the end.

We would like to point out also that in a recent paper M. F. Atiyah and M. Hopkins [AHo] introduced another type of $K$-theory, denoted by them $K_{ \pm}(X)$, which is in fact a particular case of twisted equivariant $K$-theory. More precisely, this définition of $K_{ \pm}(X)$ was already given in [K1] $\S 3.3$, 40 years ago, in terms of Clifford bundles with a group action (see 6.16 and also [K4] for the details).

This paper is not just historical. It presents the theory with a new point of view and contains some new results. We extend the Thom isomorphism to this more general setting (see also $[\mathrm{Ca}]$ ), which is important in order to relate the "ungraded" and "graded" twisted $K$-theories. We compute many interesting equivariant twisted $\mathrm{K}$-groups, complementing the basic papers [LTX], [CTX] and [AS]. For this purpose, we use the "Chern character" for finite group actions, as defined by Baum, Connes, Kuhn and Slominska [BC]Kuh][S], together with our generalized Thom isomorphism. These last computations are related to some previous ones [K4] and to the work of many authors : A. Adem and Y. Ruan [AR], P. Hu and I. Kriz [HK], J.-L. Tu and P. Xu [TX], among others. Finally, we introduce new cohomology operations which are complementary to those defined in [DK] and [AS2]

${ }^{1}$ As it was pointed out to me by J. Rosenberg, one should also add the following reference, in the spirit of [DD]: Ellen Maycock Parker, The Brauer group of graded continuous trace $\mathrm{C}^{*}$-algebras, Trans. Amer. Math. Soc. 308 (1988), no. 1, 115--132. 


\section{General plan of the paper}

Let us first recall the point of view developed in [DK], in order to describe the background material. We consider a locally trivial bundle of graded central simple complex algebras $\mathcal{A}$, i.e. modelled on $M_{2 n}(\mathbf{C})$ or $M_{n}(\mathbf{C})$ x $M_{n}(\mathbf{C})$, with the obvious gradings ${ }^{2}$. Then $\mathcal{A}$ has a welldefined class $\alpha$ in the group $\operatorname{GBr}(X)$ (as introduced above). On the other hand, one may consider the category of " $\mathcal{A}$-bundles", whose objects are vector bundles provided with an $\mathcal{A}$ module structure (fibrewise). We call this category $E^{\mathcal{A}_{(X)}}$; the graded objects of this category are vector bundles which are modules over $\mathcal{A} \otimes C^{0,1}$, where $C^{0,1}$ is the Clifford algebra $\mathbf{C} \times \mathbf{C}=\mathbf{C}[x] /\left(x^{2}-1\right)$. The group $K^{\alpha}(X)$ is now defined as the Grothendieck group of the forgetful functor

$$
E^{\mathcal{A} \otimes C^{0,1}}(X) \rightarrow E^{\mathcal{A}}(X)
$$

We refer the reader to [K1] for the definition of this group which generalizes the usual Grothendieck group of a category. For our purpose, we make it quite explicit at the end of $\S 1$, using the concept of "grading".

Despite its algebraic simplicity, the previous definition of $K^{\alpha}(X)$ is not quite satisfactory for various reasons. For instance, it is not clear how to define in a simple way a "cup-product"

$$
K^{\alpha}(X) \text { х } K^{\alpha^{\prime}}(X) \rightarrow K^{\alpha+\alpha^{\prime}}(X)
$$

as mentioned earlier (even if $\alpha$ and $\alpha^{\prime}$ are in the much smaller group $\mathbf{Z} / 2$ ).

To correct this defect, a second definition may be given in terms of Fredholm operators in a Hilbert space. More precisely, we consider graded Hilbert bundles $E$ which are also graded $\mathcal{A}$ modules in an obvious sense, together with a continuous family of Fredholm operators

with the following properties :

$$
D: E \rightarrow E
$$

1) $D$ is self-adjoint of degree one

2) $D$ commutes with the action of $\mathcal{A}$ (in the graded sense)

One gets an abelian semi-group from the homotopy classes of such couples $(E, D)$, with the addition rule

$$
(E, D)+\left(E^{\prime}, D^{\prime}\right)=\left(E \oplus E^{\prime}, D \oplus D^{\prime}\right)
$$

The associated group gives the second definition of $K^{\alpha}(X)$ which is equivalent to the first one (see [DK] p. 18 and [K2] p. 88).

With this new viewpoint, the cup-product alluded to above becomes obvious. It is defined by the following formula :

$$
(E, D) \cup\left(E^{\prime}, D^{\prime}\right)=\left(E \widehat{\otimes} E^{\prime}, D \widehat{\otimes} 1+1 \widehat{\otimes} D^{\prime}\right)
$$

2 up to graded Morita equivalence. 
where the symbol $\widehat{\otimes}$ denotes the graded tensor product of bundles or morphisms.

For simplicity's sake, we have only considered complex $K$-theory. We could as well study the real case : one has to replace $\operatorname{GBr}(\mathrm{X})$ by

$$
\operatorname{GBr} O(X)=\mathbf{Z} / 8 \times H^{1}(X ; \mathbf{Z} / 2) \times H^{2}(X ; \mathbf{Z} / 2)
$$

If we take for the coefficient system $\alpha=n$ to be in $\mathbf{Z} / 8$, we get the usual groups $K O^{n}(X)$ as defined using Clifford algebras in [K1] and [K2] p. 88 (these groups being written $\bar{K}^{\mathrm{n}}$ in the later reference).

In this paper, we essentially follow the plan above, but with bundles of "infinite dimension" as we shall explain later on. As a matter of fact, all the technical tools are already present in [DK], [K2] and [R], for instance the Fredholm operator machinery. However, this paper is not a rewriting of these papers, since we take a more synthetic view point and have other applications in mind. For instance, the $K$-theory of Banach algebras $K_{n}(A)$ and its graded version, denoted here by $\operatorname{Gr}_{n}(A)$, are more systematically used. On the other hand, since the equivariant $K$ theory has been studied carefully in [CKRW], [LTX] and [AS], we limit ourselves to the applications in this case. One of them is the definition of operations in twisted $K$-theory in the graded and ungraded situations.

Here are the contents of the paper :

1. $K$-theory of $\mathbf{Z} / 2$-graded Banach algebras

2. Ungraded twisted $K$-theory in the finite and infinite-dimensional cases

3. Graded twisted $K$-theory in the finite and infinite-dimensional cases

4. The Thom isomorphism

5. General equivariant $K$-theory

6. Some computations in the equivariant case

7. Operations in twisted $K$-theory.

Aknowledgments. I would like to thank A. Adem, A. Carey, P. Hu, I. Kriz, C. Leruste, V. Mathai, J. Rosenberg, J.-L. Tu and P. Xu for their remarks and suggestions after various drafts of this paper. 


\section{1. $K$-theory of $Z / 2$-graded Banach algebras.}

1.1. Higher $K$-theory of real or complex Banach algebras $A$ is well-known (cf. [K1] or [B] for instance). Starting from the usual Grothendieck group $K(A)=K_{0}(A)$, there are many equivalent ways to define "derived functors" $K_{n}(\mathrm{~A})$, for $n \in \mathbf{Z}$, such that any exact sequence of Banach algebras

$$
0 \rightarrow A^{\prime} \rightarrow A \rightarrow A^{\prime \prime} \rightarrow 0
$$

induces an exact sequence of abelian groups

$$
\ldots \rightarrow K_{n+1}(A) \rightarrow K_{n+1}\left(A^{\prime \prime}\right) \rightarrow K_{n}\left(A^{\prime}\right) \rightarrow K_{n}(A) \rightarrow K_{n}\left(A^{\prime \prime}\right) \rightarrow \ldots
$$

Moreover, by Bott periodicity, these groups are periodic of period 2 in the complex case and 8 in the real case.

1.2. The $K$-theory of $\mathbf{Z} / 2$-graded Banach algebras $A$ (in the real or complex case) is less wellknown $^{3}$ and for the purpose of this paper we shall recall its definition which is already present but not systematically used in [K1] and [DK]. We first introduce $C^{p, q}$ as the Clifford algebra of $\mathbf{R}^{p+q}$ with the quadratic form

$$
-\left(x_{1}\right)^{2}-\ldots-\left(x_{p}\right)^{2}+\left(x_{p+1}\right)^{2}+\ldots+\left(x_{p+q}\right)^{2}
$$

It is naturally $\mathbf{Z} / 2$-graded. If $A$ is an arbitrary $\mathbf{Z} / 2$-graded Banach algebra, $A^{p, q}$ is the graded tensor product $A \otimes C^{p, q}$. For $A$ unital, we now define the graded $K$-theory of $A$ (denoted by $\operatorname{Gr} K(A))$ as the $K$-theory of the forgetful functor :

$$
\phi: \mathcal{P}\left(A^{0,1}\right) \rightarrow \mathcal{P}(A)
$$

(see [K1] or 1.4 for a concrete definition). Here $\mathcal{P}(A)$ denotes in general the category of finitely generated projective left $A$-modules. One may remark that the objects of $\mathcal{P}\left(A^{0,1}\right)$ are graded objects of the category $\mathcal{P}(A)$. The functor $\phi$ simply "forgets" the grading. One should also notice that $\operatorname{GrK}\left(A^{p, q}\right)$ is naturally isomorphic to $\operatorname{Gr} K\left(A^{p+1, q+1}\right)$, since $A$ p,q is Morita equivalent to $A^{p+1, q+1}$ ) (in the graded sense).

If $A$ is not unital, we define $\operatorname{Gr} K(A)$ by the usual method, as the kernel of the augmentation map

$$
\operatorname{GrK}\left(A^{+}\right) \rightarrow \operatorname{GrK}(k)
$$

where $A^{+}$is the $k$-algebra $A$ with a unit added $(k=\mathbf{R}$ or $\mathbf{C}$, according to the theory, with the trivial grading).

1.3. There is a "suspension functor" on the category of graded algebras, associating to $A$ the graded tensor product $A^{0,1}=A \otimes C^{0,1}$. One of the fundamental results ${ }^{4}$ in [K1] p. 210 is the fact that this suspension functor is compatible with the usual one : in other words, we have a well-defined isomorphism

3 This is of course included in the general KK-theory of Kasparov which was introduced later than our basic references [K1] and [DK].

${ }^{4}$ Strictly speaking, one has to replace the category $C^{p, q}$ in [K1] with an arbitrary graded category. However, the proof of theorem 2.2.2 easily extends to this case (see also [W]). 


$$
\operatorname{GrK}\left(A^{0,1}\right) \cong \operatorname{GrK}(A(\mathbf{R}))
$$

where $A(T)$ denotes in general the algebra of continuous maps $f(t)$ on the locally compact space $T$ with values in $A$, such that $f(t) \rightarrow 0$ when $t$ goes to infinity. As a consequence, we have an isomorphism between the following groups (for $n \geq 0$ ) :

$$
\operatorname{GrK}\left(A^{0, n}\right) \cong \operatorname{GrK}\left(A\left(\mathbf{R}^{n}\right)\right)
$$

which we call $\operatorname{Gr} K_{n}(A)$. More generally, we put $\operatorname{Gr} K_{n}(A)=\operatorname{Gr} K\left(A^{p, q}\right)$ for $q-p=n \in \mathbf{Z}$. These groups $G r K_{n}$ satisfy the same exactness property as the groups $K_{n}(A)$ above, from which they are naturally derived. They are of course linked with them by the following exact sequence (for all $n \in \mathbf{Z})$ :

$$
K_{n+1}\left(A \widehat{\otimes} C^{0,1}\right) \rightarrow K_{n+1}(A) \rightarrow G r K_{n}(\mathrm{~A}) \rightarrow K_{n}\left(A \widehat{\otimes} C^{0,1}\right) \rightarrow K_{n}(A)
$$

In particular, if we start with an ungraded Banach algebra $A$, we see that Bott periodicity follows from these previous considerations, thanks to the periodicity of Clifford algebras up to graded Morita equivalence : this was the main theme developed in [K1], in order to give a more conceptual proof of the periodicity theorems.

1.4. When $A$ is unital, it is technically important to describe the group $\operatorname{Gr} K(A)$ in a concrete way. If $E$ is an object of $\mathcal{P}(A)$, a grading of $E$ is given by an involution $\varepsilon$ which commutes (resp. anticommutes) with the action of the elements of degree 0 (resp. 1) in $A$. In this way, $E$ with a grading $\varepsilon$ may be viewed as a module over the algebra $A \widehat{\otimes} C^{0,1}$. We now consider triples $\left(E, \varepsilon^{1}, \varepsilon^{2}\right)$, where $\varepsilon^{1}$ and $\varepsilon^{2}$ are two gradings of $E$. The homotopy classes of such triples obviously form a semi-group. Its quotient by the semi-group of "elementary" triples (i.e. such that $\varepsilon^{1}=\varepsilon^{2}$ ) is isomorphic to $\operatorname{GrK}(A)$.

\section{Ungraded twisted K-theory in the finite and infinite-dimensional cases.}

2.1. Let $X$ be a compact space and let us consider bundles of algebras $\mathcal{A}$ with fiber $M_{n}(\mathbf{C})$. As was shown by Serre [G], such bundles are classified by Cech cocycles (up to Cech coboundaries):

$$
g_{j i}: U_{i} \cap U_{j} \rightarrow P U(n)
$$

where $P U(n)$ is $U(n) / S^{1}$, the projective unitary group. In other words, the bundle of algebras $\mathcal{A}$ may be obtained by glueing together the bundles of $\mathrm{C}^{*}$-algebras $\left(U_{i} \mathrm{x} M_{n}(\mathbf{C})\right)$, using the transition functions $g_{j i}$ [Note that $U(n)$ acts on $M_{n}(\mathbf{C})$ by inner automorphisms and therefore induces an action of $P U(n)$ on the algebra $M_{n}(\mathbf{C})$ ]. The Brauer group of $X$ denoted by $\operatorname{Br}(X)$ is obtained as the quotient of this semi-group of bundles (via the tensor product) by the following equivalence relation: $\mathcal{A}$ is equivalent to $\mathcal{A}$ ' iff there exist vector bundles $V$ and $V^{\prime}$ such that the bundles of algebras $\mathcal{A} \otimes E N D(V)$ and $\mathcal{A}^{\prime} \otimes E N D\left(V^{\prime}\right)$ are isomorphic. It was proved by Serre [G] that $\operatorname{Br}(X)$ is naturally isomorphic to the torsion subgroup of $H^{3}(X ; \mathbf{Z})$.

The Serre-Swan theorem (cf. [K1] for instance) may be easily translated in this situation to show that the category of finitely generated $\mathcal{A}$-module bundles $E$ (as in $[\mathrm{DK}]$ ) is equivalent to the category $\mathcal{P}(A)$ of finitely generated projective modules over $A=\Gamma(X, \mathcal{A})$, the algebra of continuous sections of the bundle $\mathcal{A}$. The key observation for the proof is that $E$ is a direct 
factor of a "trivial" $\mathcal{A}$-bundle ; this is easily seen with finite partitions of unity, since $X$ is compact. One should notice that if $\mathcal{A}$ is equivalent to $\mathcal{A}$ ' the associated categories $\mathcal{P}(A)$ and $\mathcal{P}\left(A^{\prime}\right)$ are equivalent.

2.2. DEFINITION. The ungraded ${ }^{5}$ twisted $K$-theory $K^{(\mathcal{A})}(X)$ is by definition the $K$-theory of

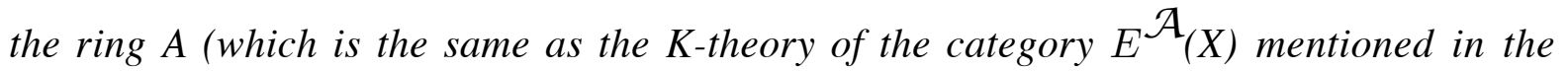
introduction). By abuse of notation, we shall simply call it $K(\mathcal{A})$. We also define $K_{n}(\mathcal{A})$ as the $K_{n^{-}}$group of the Banach algebra $\Gamma(X, \mathcal{A})$. It only depends on the class of $\mathcal{A}$ in $\operatorname{Br}(X)=$ $\operatorname{Tors}\left(H^{3}(X ; \mathbf{Z})\right)$.

2.3. The key observation made by $J$. Rosenberg $[R]$ is the following : we can "stabilize" the situation (in the $\mathrm{C}^{*}$-algebra sense), i.e. embed $M_{n}(\mathbf{C})$ into the algebra of compact operators $\mathcal{K}$ in a separable Hilbert space $H$, thanks to the split inclusion of $\mathbf{C}^{n}$ in $l^{2}(\mathbf{N})$. Now, a bigger group $P U(H)=U(H) / S^{1}$ is acting on $\mathcal{K}$ by inner automorphisms. If we take a Cech cocycle

$$
g_{j i}: U_{i} \cap U_{j} \rightarrow P U(H)
$$

we may use it to construct a bundle $\mathcal{A}$ of (non unital) $\mathrm{C}^{*}$-algebras with fiber $\mathcal{K}$.

Let us now consider the commutative diagram

$$
\begin{aligned}
& S^{1} \rightarrow S^{1} \\
& \downarrow \quad \downarrow \\
& U(n) \rightarrow U(H) \\
& \downarrow \quad \downarrow \\
& P U(n) \rightarrow P U(H)
\end{aligned}
$$

Thanks to Kuiper's theorem [Ku], we remark that the classifying space of $U(H)$ is contractible. Therefore, the classifying space $B P U(H)$ of the topological group $P U(H)$, is a nice model of the Eilenberg-Mac Lane space $K(\mathbf{Z}, 3)$ (compare with the well-known paper of Dixmier and Douady [DD]). Moreover, if we start with a finite-dimensional algebra bundle $\mathcal{A}$ over $X$ with fiber $M_{n}(\mathbf{C})$, the diagram above shows how to associate to $\mathcal{A}$ another bundle of algebras $\mathcal{A}$ ' with fiber $\mathcal{K}$, together with a $\mathrm{C}^{*}$-inclusion from $\mathcal{A}$ to $\mathcal{A}$ '. We note that the invariant $W_{3}(\mathcal{A})$ in $\operatorname{Br}(X)=\operatorname{Tors}\left(H^{3}(X ; \mathbf{Z})\right)$ defined in $[\mathrm{G}]$ is simply induced by the classifying map from $X$ to $B P U(H)$ (which factors through $B P U(n)$ ). In this finite example, it is an $n$-torsion class since one has another commutative diagram

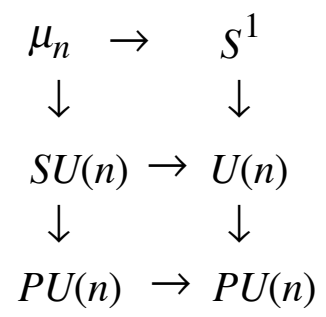

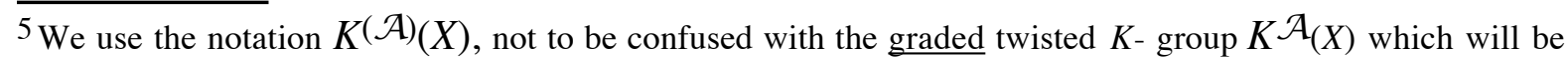
defined in the next section. 
2.4. THEOREM. The inclusion from $\mathcal{A}$ to $\mathcal{A}$ ' induces an isomorphism

$$
K_{r}(\mathcal{A})=K_{r}(\Gamma(X, \mathcal{A})) \rightarrow K_{r}\left(\Gamma\left(X, \mathcal{A}^{\prime}\right)\right)=K_{r}\left(\mathcal{A}^{\prime}\right)
$$

where $K_{r}$ is the classical topological $K$-theory of $C^{*}$-algebras.

Proof. The proof is classical for a trivial algebra bundle, since $\mathbf{C}$ is Morita equivalent to $\mathcal{K}$ (in the $C^{*}$-algebra sense). It extends to the general case by a no less classical Mayer-Vietoris argument.

2.5. DEFINITION. Let now $\mathcal{A}$ be an algebra bundle with fiber $\mathcal{K}$ on a compact space $X \underline{\text { with }}$ structural group $P U(H)$. We define $K^{(\mathcal{A})}(X)$ (also denoted by $K(\mathcal{A})$ ) as the $K$-theory of the (non unital) Banach algebra $\Gamma(X, \mathcal{A})$. This $K$-theory only depends of the class $\alpha$ of $\mathcal{A}$ in $H^{3}(X ; \mathbf{Z})$ and we shall also call it $K^{(\alpha)}(X)$ [Due to 2.4 , this is a genereralization of definition $2.2]$.

2.6. Before treating the graded case in the next section, we would like to give an equivalent definition of $K(\mathcal{A})$ in terms of Fredholm operators, as was done in [DK] for the torsion elements in $H^{3}(X ; \mathbf{Z})$ and, later on, in [AS] for the general case. The basic idea is to remark that $P U(H)$ acts not only on the $\mathrm{C}^{*}$-algebra of compact operators in $H$, but also on the ring of bounded operators $\operatorname{End}(H)$ and on the Calkin algebra $\operatorname{End}(H) / \mathcal{K}$ (with the norm topology). Let us call $\mathcal{B}$ the algebra bundle with fiber $\operatorname{End}(H)$ associated to the cocycle defined in 2.1 and $\mathcal{B} / \mathcal{A}$ the quotient algebra bundle. Therefore, we have an exact sequence of $\mathrm{C}^{*}$-algebra bundles

$$
0 \rightarrow \mathcal{A} \rightarrow \mathcal{B} \rightarrow \mathcal{B} / \mathcal{A} \rightarrow 0
$$

which induces an exact sequence for the associated rings of sections (thanks to a partition of unity again)

$$
0 \rightarrow \Gamma(X, \mathcal{A}) \rightarrow \Gamma(X, \mathcal{B}) \stackrel{\pi}{\longrightarrow} \Gamma(X, \mathcal{B} / \mathcal{A})) \rightarrow 0
$$

If $\mathcal{B}$ is trivial, it is well-known that the algebra of continuous maps from $X$ to $\operatorname{End}(H)$ has trivial $K_{n}$-groups because this algebra is flabby ${ }^{6}$. By a Mayer-Vietoris argument, it follows that $K_{n}(\Gamma(X, \mathcal{B}))$ is also trivial. Therefore the connecting homomorphism

$$
K_{1}(\mathcal{B} / \mathcal{A})=K_{1}(\Gamma(X, \mathcal{B} / \mathcal{A})) \rightarrow K_{0}(\Gamma(X, \mathcal{A})) \cong K^{(\mathcal{A})}(X)=K(\mathcal{A})
$$

is an isomorphism, a well-known observation in index theory.

2.7. Let us now consider the elements of $\mathcal{B}$ which map onto $(\mathcal{B} / \mathcal{A})^{*}$ via the map $\pi$. These elements form a bundle of Fredholm operators on $H$ (the twist comes from the action of $P U(H)$ ). This subbundle of $\mathcal{B}$ will be denoted by $\mathcal{F r e d}(H)$. Therefore, we have a principal fibration

$$
\Gamma(X, \mathcal{A}) \rightarrow \Gamma(X, \mathcal{F r e d}(H)) \rightarrow \Gamma\left(X,(\mathcal{B} / \mathcal{A})^{*}\right)
$$

\footnotetext{
${ }^{6}$ A unital Banach algebra $\Lambda$ is called flabby if there exists a continuous functor $\tau$ from $\mathcal{P}(A)$ to itself such that $\tau+\mathrm{Id}$ is isomorphic to $\tau$. For instance, $A=\operatorname{End}(H)$ is flabby since $\mathcal{P}(A)$ is equivalent to the category $C$ of Hilbert spaces which are isomorphic to direct factors in $H ; \tau$ is then defined by the infinite Hilbert $\operatorname{sum} \tau(E)=$ $E \oplus \ldots \oplus E \oplus \ldots$
} 
with contractible fiber the Banach space $\Gamma(X, \mathcal{A})$ (this fibration admits a local section thanks to Michael's theorem [Mi]). Therefore, the space of sections of $\mathcal{F r e d} \hat{h}(H)$ has the homotopy type of $\Gamma\left(X,(\mathcal{B} / \mathcal{A})^{*}\right)$. In particular, the path components are in bijective correspondence via the map $\pi$. The following theorem is a generalization of a well-known theorem of Atiyah and Janich $[\mathrm{A} 1][\mathrm{J}]$ :

2.8. THEOREM [AS]. The set of homotopy classes of continuous sections of the fibration

is naturally isomorphic to $K^{(\mathcal{A})}(X)$.

$$
\text { Fredh }(H) \rightarrow X
$$

Proof. As we have seen above, the two spaces $\Gamma(X, \mathcal{F r e d} h(H))$ and $\Gamma\left(X,(\mathcal{B} / \mathcal{A})^{*}\right)$ have the same homotopy type. On the other hand, it is a well-known consequence of Kuiper's theorem $[\mathrm{Ku}]$ than the (non unital) ring map $\Gamma(X, \mathcal{B} / \mathcal{A}) \rightarrow \Gamma\left(X, \mathrm{M}_{\mathrm{r}}(\mathcal{B} / \mathcal{A})\right)$ induces a bijection between the path components of the associated groups of invertible elements (see for instance [K5] p. 93). Therefore, $\pi_{0}(\Gamma(X, \mathcal{F r e d} h(H)))$ may be identified with

$$
\lim _{\vec{r}} \pi_{0}\left(\Gamma\left(X, G L_{r}(\mathcal{B} / \mathcal{A})\right)\right)=K_{1}(\mathcal{B} / \mathcal{A})
$$

and therefore with $K(\mathcal{A})$, as we already mentioned in 2.6.

2.9. Remark. We may also consider the following "stabilized" bundle

$$
\text { Fred }_{\mathrm{s}}(H)=\lim _{\vec{n}} \mathcal{F r e d h}\left(H^{n}\right)
$$

and, without Kuiper's theorem, prove almost in the same way that the connected component of the space of sections of this bundle is also isomorphic to $K^{(\mathcal{A})}(X)$.

2.10. There is an obvious ring homomorphism (since the Hilbert tensor product $H \otimes H$ is isomorphic to $H$ )

$$
\mathcal{K} \otimes \mathcal{K} \rightarrow \mathcal{K}
$$

If $\mathcal{A}$ and $\mathcal{A}$ ' are bundles of algebras on $\mathrm{X}$ modelled on $\mathcal{K}$, we may use this homomorphism to get a new algebra bundle on $\mathrm{X}$, which we denote by $\mathcal{A} \otimes \mathcal{A}$ ' From the cocycle point of view, we have a commutative diagram, where the top arrow is induced by complex multiplication

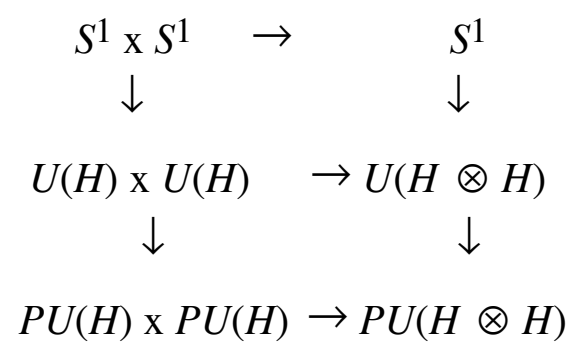

It follows that $W_{3}\left(\mathcal{A} \otimes \mathcal{A}^{\prime}\right)=W_{3}(\mathcal{A})+W_{3}\left(\mathcal{A}^{\prime}\right)$ in $H^{3}(X ; \mathbf{Z})$ and one gets a "cup-product"

$$
K^{(\alpha)}(X) \times K^{\left(\alpha^{\prime}\right)}(X) \rightarrow K^{\left(\alpha+\alpha^{\prime}\right)}(X)
$$

This is a particular case of a "graded cup-product" which will be introduced in the next section. 


\section{Graded twisted $K$-theory in the finite and infinite-dimensional cases.}

3.1. We are going to change our point of view and now consider $\mathbf{Z} / 2$-graded finite-dimensional algebras which are central and simple (in the graded sense). We are only interested in the complex case. The real case is treated with great details in [DK] and does not seem to generalize to the infinite-dimensional framework ${ }^{7}$.

In the complex case, there are just two "types" of graded algebras (up to Morita equivalence ${ }^{8}$ ) which are $R=\mathbf{C}$ and $\mathbf{C} \times \mathbf{C}=\mathbf{C}[x] /\left(x^{2}-1\right)$. For a type $R$ of algebra, the graded inner automorphisms of $A=R \widehat{\otimes} \operatorname{End}(V \oplus W)$ may be given by either an element of degree 0 or an element of degree 1 in $A^{*}$. This gives us an augmentation (whose kernel is denoted by $\left.\operatorname{Aut}^{0}(A)\right)$ :

$$
\operatorname{Aut}(A) \rightarrow \mathbf{Z} / 2
$$

Therefore, for bundles of $\mathbf{Z} / 2$-graded algebras modelled on $A$, we already get an invariant in $H^{1}(X ; \mathbf{Z} / 2)$, called the "orientation" of $A$ and which may be represented by a line bundle. A typical example is the (complexified) Clifford bundle $C(V)$, associated to a real vector bundle $V$ of rank $n$. Its orientation invariant is the first Stiefel-Whitney class associated to $V$ (cf. [DK]). Note that the type $R$ of $C(V)$ is $\mathbf{C}$ if $n$ is even and $\mathbf{C} \times \mathbf{C}$ if $n$ is odd ${ }^{9}$.

3.2. For the second invariant, let us start with $M_{2 n}(\mathbf{C})$ as a basic graded algebra to fix the ideas and let us put a $C^{*}$-algebra metric on $A$. We have an exact sequence of groups as in the ungraded case (where $\left.P U^{0}(2 n)=\mathrm{P} U(2 n) \cap A u t^{0}(A)\right)$

$$
1 \rightarrow S^{1} \rightarrow U(n) \text { x } U(n) \rightarrow P U^{0}(2 n) \rightarrow 1
$$

Therefore, a bundle with structural group $P U^{0}(2 n)$ also has a class in $H^{2}\left(X ; \boldsymbol{S}^{1}\right)=H^{3}(X ; \boldsymbol{Z})$ which is easily seen to have order $n$ as in the ungraded case. A similar argument holds if the graded algebra is $M_{n}(\boldsymbol{C})$ x $M_{n}(\boldsymbol{C})$. It follows that the "graded Brauer group" $G B r(X)$ is

$$
\operatorname{GBr}(X)=\mathbf{Z} / 2 \times H^{1}(X ; \mathbf{Z} / 2) \times \operatorname{Tors}\left(H^{3}(X ; \mathbf{Z})\right)
$$

as already quoted in the introduction (see [DK] for the explicit group law on $\operatorname{GBr}(X)$ ). If $\mathcal{A}$ is a bundle of $\mathbf{Z} / 2$-graded finite-dimensional algebras, we define the graded twisted $K$-theory $K^{\mathcal{A}}(X)$ as the graded $K$-theory of the graded algebra $\Gamma(X, \mathcal{A})$ as recalled in Section 1 . This definition only depends on the class of $\mathcal{A}$ in $\operatorname{GBr}(X)$. We recover the definition in [DK] by using again the Serre-Swan theorem as in $\S 2$. For instance, if we consider the bundle of (complex) Clifford algebras $C(V)$, associated to a real vector bundle $V$, the invariants we get are $w_{1}(V)$ and $W_{3}(V)$, as quoted in the introduction. If these invariants are trivial, the bundle $V$ is

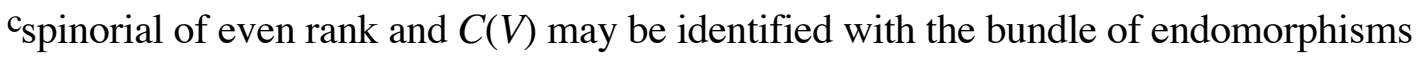

$\operatorname{End}\left(S^{+} \oplus S^{-}\right)$, where $S^{+}$and $S^{-}$are the even and odd "spinors" associated to the Spincstructure.

\footnotetext{
7 This is not quite true if we work in the context of "Real" $K$-theory in the sense of Atiyah [A1]. We shall not consider this generalization here, although it looks interesting in the light of "equivariant twisted $K$-theory" as we shall show in $\S 5$.

8 This means that we are allowed to take the graded tensor product with $\operatorname{End}(V \oplus W)$ with the obvious grading.

9 If $V$ is oriented and even dimensional for instance, this does not imply that $C(V)$ is a bundle of graded algebras of type $M_{2}(\Lambda)$ for a certain bundle of ungraded algebras $\Lambda$. However, for suitable vector bundles $V$ and $W$, this is the case for the graded tensor product $C(V) \widehat{\otimes} \operatorname{End}(V \oplus W)$.
} 
3.3. In order to define graded twisted $K$-theory in the infinite-dimensional case, we follow the same pattern as in $\S 2$. For instance, let us take a graded bundle of algebras $\mathcal{A}$ modelled on $M_{2}(\mathcal{K})$ : it has 2 invariants, one in $H^{1}(X ; \mathbf{Z} / 2)$, the other in $H^{3}(X ; \mathbf{Z})$ (and not just in the torsion of this group). We then define $K^{\mathcal{A}}(X)$ as the graded $K$-theory of the graded algebra $\Gamma(X, \mathcal{A})$, according to $\S 1$. The same definition holds for bundles of graded algebras modelled on $\mathcal{K}$ x $\mathcal{K}=\mathcal{K}[x] /\left(x^{2}-1\right)=\mathcal{K} \oplus \mathcal{K} x$.

If $C^{0,1}$ is the Clifford algebra $\mathbf{C} \times \mathbf{C}$ with its usual graded structure, the general results of $\S 1$ show that the group $K^{\mathcal{A}}(X)$ fits into an exact sequence :

$$
\left.K_{1}\left(\mathcal{A} \widehat{\otimes} C^{0,1}\right)(X) \rightarrow K_{1}(\mathcal{A})(X) \rightarrow K^{\mathcal{A}}(X) \rightarrow K^{(\mathcal{A} \otimes} \widehat{\otimes} C^{0,1}\right)(X) \rightarrow K^{(\mathcal{A})}(X)
$$

where $K_{\mathrm{i}}{ }^{(\mathcal{A})}(X)$ denotes in general the $K_{\mathrm{i}}$-group of the Banach algebra $\Gamma(X, \mathcal{A})$.

3.4. Let us now assume that $\mathcal{A}$ is oriented modelled on $M_{2}(\mathcal{K})$. We are going to show that $\mathcal{A}$ may be written as $M_{2}\left(\mathcal{A}^{\prime}\right)$, with the obvious grading, $\mathcal{A}^{\prime}$ being an ungraded bundle of algebras modelled on $\mathcal{K}$. For this purpose, we write the commutative diagram

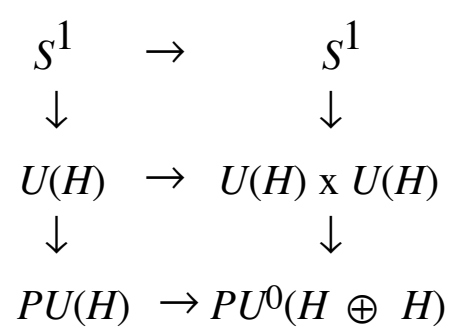

where the first horizontal map is the identity and the others are induced by the diagonal. This shows that $H^{1}(X ; P U(H)) \cong H^{1}\left(X ; P U^{0}(H \oplus H)\right)$, which is equivalent to saying that $\mathcal{A}$ may be written as $M_{2}\left(\mathcal{A}^{\prime}\right)$ for a certain bundle of algebras $\mathcal{A}$ '.

Therefore, $\mathcal{A} \otimes C^{0,1}$ is Morita equivalent to $\mathcal{A}^{\prime} \times \mathcal{A}^{\prime}$ and $K^{\mathcal{A}}(X)$ is the $K$-theory of the ring homomorphism

$$
\mathcal{A}^{\prime} \times \mathcal{A}^{\prime} \rightarrow M_{2}\left(\mathcal{A}^{\prime}\right)
$$

defined by $(a, b) \mapsto\left(\begin{array}{ll}a & 0 \\ 0 & b\end{array}\right)$ (no grading). We should also note that $\mathcal{A}$ ' is Morita equivalent to $\mathcal{A}$ as an ungraded bundle of algebras. Since the usual $K$-theory (resp. graded $K$-theory) is invariant under Morita equivalence (resp. graded Morita equivalence), the previous considerations lead to the following theorem :

3.5. THEOREM. Let $\mathcal{A}$ be an oriented bundle of graded algebras modelled on $M_{2}(\mathcal{K})$. Then $K^{\mathcal{A}}(X)$ is isomorphic to $K^{(\mathcal{A})}(X)$ via the identification above.

3.6. The same method may be applied in the case when $\mathcal{A}$ is an oriented bundle of graded algebras modelled on $\mathcal{K}_{\mathrm{x}} \mathcal{K}=\mathcal{K}[x] /\left(x^{2}-1\right)$. The graded oriented automorphisms of $\mathcal{K} \mathrm{x} \mathcal{K}$ induced by $P U(H \oplus H)$ are diagonal matrices of type 


$$
\left(\begin{array}{ll}
a & 0 \\
0 & a
\end{array}\right)
$$

This shows that $\mathcal{A}$ is isomorphic to $\mathcal{A}^{\prime} \mathrm{x} \mathcal{A}^{\prime}$ and $\mathcal{A} \otimes \mathrm{C}^{0,1}$ is isomorphic to $\mathrm{M}_{2}\left(\mathcal{A}^{\prime}\right)$. Therefore, $\mathrm{K}^{\mathcal{A}}(\mathrm{X})$ is the Grothendieck group of the ring homomorphism $\mathcal{A}^{\prime} \rightarrow \mathrm{M}_{2}\left(\mathcal{A}^{\prime}\right)$, defined by

$$
a \mapsto\left(\begin{array}{cc}
a & 0 \\
0 & a
\end{array}\right)
$$

Hence we have the following theorem, analogous to 3.5 :

3.7. THEOREM. Let $\mathcal{A}$ be an oriented bundle of graded algebras modelled on $\mathcal{K} x \mathcal{K}$. Then $\mathcal{A}$ is isomorphic to $\mathcal{A}^{\prime} x \mathcal{A}^{\prime}$ and the group $K^{\mathcal{A}}(X)$ is isomorphic to $K_{1}\left(\mathcal{A}^{\prime}\right)$ via the identification above.

3.8. Remark. One may notice that if $\mathcal{A}$ is a bundle of oriented graded algebras modelled on $M_{2}(\mathcal{K})$, the associated bundle with fiber $M_{2}\left(\mathcal{K}^{+}\right)$has a section $\varepsilon$ of degree 0 and of square 1 , which commutes (resp. anticommutes) with the elements of degree 0 (resp. 1); it is simply defined by the matrix

$$
\left(\begin{array}{cc}
1 & 0 \\
0 & -1
\end{array}\right)
$$

3.9. Finally, we would like to give an equivalent definition of $K^{\mathcal{A}}(X)$ in terms of Fredholm operators as in $\S 2$. This was done in $[\mathrm{DK}]$ if the class of $\mathcal{A}$ belongs to the torsion group of $H^{3}(X ; \mathbf{Z})$ and later on in $[\mathrm{AS}]$ for the general case. We shall give a simplified treatment here, using again the $K$-theory of graded Banach algebras.

Following the general notations of $\S 2$, we have an exact sequence of bundles of graded Banach algebras

$$
0 \rightarrow \mathcal{A} \rightarrow \mathcal{B} \rightarrow \mathcal{B} / \mathcal{A} \rightarrow 0
$$

Since the graded $K$-groups of $\mathcal{B}$ are trivial, we see as in 2.6 that $\operatorname{Gr} K(\Gamma(X, \mathcal{A}))$ is isomorphic to $\operatorname{GrK}_{1}(\Gamma(X, \mathcal{B} / \mathcal{A}))$.

In order to shorten the notations, we denote by $B$ the graded Banach algebra $\Gamma(X, \mathcal{B})$, by $\Lambda$ the graded Banach algebra $\Gamma(X, \mathcal{B} / \mathcal{A})$ and by $\phi$ the surjective map $B \rightarrow \Lambda$. The following lemma ${ }^{10}$ may be proved in the same way as in [K2] p. 78 :

3.10. LEMMA. Any element of $\operatorname{Gr}_{1}(\Lambda)$ may be written as the homotopy class of a couple $(E, \varepsilon)$ where $E$ is a free graded B-module and $\varepsilon$ is a grading of degree one of the associated $\Lambda$ module (see 1.4 for the definition of a grading).

${ }^{10}$ It might be helpful for a better understanding to notice that the category of finitely generated free $\operatorname{End}(H)$ modules is equivalent to the category of Hilbert spaces $H^{n}$ for $n \in N$. This "local" situation is twisted by the group $P U(H)$. 
3.11. By the well-known dictionary between modules and bundle theory, we may view $\varepsilon$ as a grading of a suitable bundle $E$ of free $E n d(H) / \mathcal{K}$-modules. By spectral theory, we may also assume that $\varepsilon$ is self-adjoint. Finally, following [K2], we define a quasi-grading ${ }^{11}$ of $E$ as a family of Fredholm endomorphisms $D$ such that

1) $D^{*}=D$

2) $D$ is of degree one

The following theorem is the analogue in the graded case of Theorem 2.8 (cf [K2] p. 78/79).

3.12. THEOREM. The (graded) twisted $K$-group $K^{\mathcal{A}}(X)$ is the Grothendieck group associated to the semi-group of homotopy classes of couples $(E, D)$ where $E$ is a free $\mathbf{Z} / 2$ graded $\mathcal{B}$-module and $D$ is a family of Fredholm endomorphisms of $E$ which are self-adjoint and of degree 1.

3.13. Remark. Let us assume that $\mathcal{A}$ is oriented modelled on $M_{2}(\mathcal{K})$. The description above gives a Fredholm description of $\operatorname{Gr}_{1}(\mathcal{A})=K_{1}(\mathcal{A})$ : we just take the homotopy classes of sections of the associated bundle of self-adjoint Fredholm operators $\mathcal{F r e d h o l m}{ }^{*}(\mathcal{B})$ whose essential spectrum is divided into two non empty parts ${ }^{12}$ in $\mathbf{R}^{+*}$ and $\mathbf{R}^{-*}$

3.14. This Fredholm description of $K^{\mathcal{A}}(X)$ enables us to define a cup-product

$$
K^{\mathcal{A}}(\mathrm{X}) \times K^{\mathcal{A}^{\prime}}(X) \rightarrow K^{\mathcal{A} \otimes} \mathcal{A}^{\prime}(X)
$$

where $\mathcal{A} \otimes \mathcal{A}^{\prime}$ denotes the graded tensor product of $\mathcal{A}$ and $\mathcal{A}$ '. This cup-product is given by the same formula as in $[\mathrm{DK}]$ p. 19 and generalizes it:

$$
(E, D) \cup\left(E^{\prime}, D^{\prime}\right)=\left(E \widehat{\otimes} E^{\prime}, D \otimes \widehat{\otimes} 1+1 \widehat{\otimes} D^{\prime}\right)
$$

(see also [AS] p. 20 for a later reference).

3.15. To conclude this section, let us consider a locally compact space $X$ and a bundle of graded algebras $\mathcal{A}$ on $X$. For technical reasons, we assume the existence of a compact space $Z$ containing $X$ as an open subset, such that $\mathcal{A}$ extends to a bundle (also called $\mathcal{A}$ ) on $Z$. There is an obvious definition of $K^{\mathcal{A}}(X)$ as a relative term in the following exact sequence (where $T=$ $Z-X$ and $\left.\mathcal{A}^{\prime}=\mathcal{A} \otimes C^{0,1}\right)$ :

$$
K^{\mathcal{A}^{\prime}}(Z) \rightarrow K^{\mathcal{A}^{\prime}}(T) \rightarrow K^{\mathcal{A}_{(X)}} \rightarrow K^{\mathcal{A}_{(Z)}} \rightarrow K^{\mathcal{A}_{(T)}}
$$

By the usual excision theorem in topological $K$-theory, one may prove that this definition of $K^{\mathcal{A}}(X)$ is independent from the choice of $Z$.

The method described before and also in [K2] $\S 3$ shows how to generalize the definition of $K^{\mathcal{A}}(X)$ in this case : one takes homotopy classes of couples $(E, D)$ as in 3.12 , with the added assumption that the family $D$ is acyclic at infinity. In other words, there is a compact set $S \subset X$, such that $D_{x}$ is an isomorphism when $x \notin S$ (see [K2] p. 89-97 for the technical details of this approach). This Fredholm description of $K^{\mathcal{A}}(X)$ will be important in the next section for the description of the Thom isomorphism.

\footnotetext{
11 "quasi-graduation" in French.

12 There are two independent references in the non twisted case, i.e. for the usual classifying spaces of real or complex $K$-theory, which are [ASi] and [K5].
} 


\section{The Thom isomorphism in twisted $K$-theory ${ }^{13}$.}

4.1. Let $V$ be a finite-dimensional real vector bundle on a locally compact space $X$ which extends over a compactification of $X$ as was assumed in 3.15. Then the complexified Clifford bundle $C(V)$ has a well-defined class in the (graded) twisted $K$-theory of $X$. If $\mathcal{A}$ is another twist on $X$, we can consider the graded tensor product $\mathcal{A} \otimes C(V)$ and the associated group $K^{\mathcal{A} \otimes} \mathrm{C}(\mathrm{V})(X)$. As was described in 3.15 , it is the group ${ }^{14}$ associated to couples $(E, D)$ where $E$ is a graded bundle of free $\mathcal{B}$-modules and $D$ is a family of Fredholm endomorphisms which are of degree 1, self-adjoint and acyclic at $\infty$. Let us now consider the projection $\pi: X \times V \rightarrow$ $X$. For simplicity's sake, we shall often call $E, \mathcal{A}, \mathcal{B}, \ldots$ the respective pull-backs of $E, \mathcal{A}, \mathcal{B}$, ... via this projection. Since $\mathcal{B}$ and $C(V)$ are subbundles of $\mathcal{B} \otimes C(V), E$ may be provided with induced $\mathcal{B}$-module and $C(V)$-module structures.

4.2. THEOREM. Let $d(E, D)$ be an element of $K^{\mathcal{A} \circledast C(V)}(X)$ with the notations above. We define an element $t(d(E, D))$ in the group $K^{\mathcal{A}}(V)$ as $d\left(\pi^{*}(E), D^{\prime}\right)$ where $D^{\prime}$ is the family of Fredholm operators on $\pi^{*}(E)$, defined over the point $v$ in $V$ (with projection $\times$ on $X$ ) as

$$
D_{(x, v)}^{\prime}=D_{x}+\rho(v)
$$

where $\rho(v)$ denotes the action of the element $v$ of the vector bundle $V$ considered as a subbundle of $C(V)$. The homomorphism

$$
t: K^{\mathcal{A} \otimes} C(V)(X) \rightarrow K^{\mathcal{A}}(V)
$$

( $t$ for "Thom") defined by the formula above is then an isomorphism.

Proof $^{15}$. We should first notice that $V$ may be identified with the open unit ball bundle of the vector bundle $V$ and is therefore an open subset of the closed unit ball bundle of $V$. Moreover, since $V$ extends to a compactification of $X$, as well as $\mathcal{A}$, the required conditions in 3.15 for the definition of the twisted $K$-theory of $X$ and $V$ are fulfilled.

We shall now provide two different proofs of the theorem.

The first one, more elementary in spirit, consists in using a Mayer Vietoris argument which we can apply here since the two sides of the formula above behave as cohomology theories ${ }^{16}$ with respect to the base $X$. Therefore, we may assume that $\mathcal{A}$ and $V$ are trivial : this is a special case of the theorem stated in [K3] p. 211/212.

The second one is more subtle and may be generalized to the equivariant case. Let us first describe the Thom isomorphism for complex $V$. This is a slight modification of Atiyah's argument using the elliptic Dolbeault complex [A2]. More precisely, we consider the composite map

\footnotetext{
$\longdiv { 1 3 \text { See also [Ca]. } }$

${ }^{14}$ We should note that $E$ is a $\mathcal{B}$-module, not an $\mathcal{A}$-module. Nevertheless, we shall keep the notation $K^{\mathcal{A}}$.

${ }^{15}$ According to a suggestion of J. Rosenberg, it should be possible to give a proof with the $K K$-theory of Kasparov by describing an explicit inverse to the homomorphism $t$. However, $K K$-theory is out of the scope of this paper.

16 Strictly speaking, one has to "derive" the two members of the formula, which can be done since they are Grothendieck groups of graded Banach categories.
} 


$$
\phi: K^{\mathcal{A}}(X) \rightarrow K^{\mathcal{A} \otimes} C(V)(X) \stackrel{t}{\rightarrow} K^{\mathcal{A}}(V)
$$

The first map $\theta$ is the cup-product with the algebraic "Thom class" which is $A V$ provided with the classical Clifford graded module structure. This map is an isomorphism from well- known algebraic considerations (Morita equivalence). Therefore $t$ is an isomorphism if and only if $\phi$ is an isomorphism. On the other hand, $\phi$ is just the cup-product with the topological Thom class $T_{V}$ which belongs to the usual topological $K$-theory $K(V)$ of Atiyah and Hirzebruch [AH]. In order to prove that $\phi$ is an isomorphism, we may now use the exact sequence in 3.3 to reduce ourselves to the ungraded twisted case. In other words, it is enough to show that the cupproduct with $T_{V}$ induces an isomorphism

$$
\psi: K^{(\mathcal{A})}(X) \rightarrow K^{(\mathcal{A})}(V)
$$

In order to prove this last point, Atiyah defines a reverse map ${ }^{17}$

$$
\psi^{\prime}: K^{(\mathcal{A})}(\mathrm{V}) \rightarrow K^{(\mathcal{A})}(X)
$$

He shows that $\psi^{\prime} \psi=$ Id and, by an ingenious argument, deduces that $\psi \psi^{\prime}=$ Id as well !

Now, as soon as Theorem 4.2 is proved for complex $V$, the general case follows from a trick already used in [K3] p. 241 : we consider the following three Thom homomorphisms which behave "transitively":

$$
\begin{aligned}
& K^{\mathcal{A} \otimes} C(V) \widehat{\otimes} C(V) \widehat{\otimes} C(V)(X) \rightarrow K^{\mathcal{A} \otimes} C(V) \widehat{\otimes} C(V)(V) \rightarrow K^{\mathcal{A} \otimes} C(V)(V \oplus V) \\
& \rightarrow K^{\mathcal{A}}(V \oplus V \oplus V)
\end{aligned}
$$

We know that the composites of two consecutive arrows are isomorphisms since $V \oplus V$ carries a complex structure. It follows that the first arrow is an isomorphism, which is essentially the stated theorem (using Morita equivalence again).

4.3. Let $\mathcal{A}$ be a graded twist. As we have seen before, it has two invariants in $H^{1}(X ; \boldsymbol{Z} / 2)$ and in $H^{3}(X ; \boldsymbol{Z})$. The first one provides a line bundle $L$ in such a way that the graded tensor product $\mathcal{A}_{1}=\mathcal{A} \otimes C(L)$ is oriented. From the Thom isomorphism and the considerations in 3.5/7, we deduce the following theorem which gives the relation between the ungraded and graded twisted $K$-groups.

4.4. THEOREM. Let $\mathcal{A}$ be a graded twist of type $M_{2}(\mathcal{K})$ and $\mathcal{A}{ }_{1}=\mathcal{A} \otimes C(L)$, where L is the orientation bundle of $\mathcal{A}$. Then $\mathcal{A}_{1}$ may be written as $\mathcal{A}_{2} \times \mathcal{A}_{2}$ where $\mathcal{A}_{2}$ is ungraded of type $\mathcal{K}$. Therefore, we have the following isomorphisms

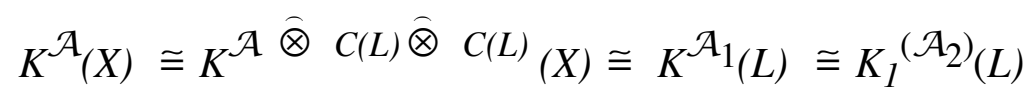

Let $\mathcal{A}$ be a graded twist of type $\mathcal{K} \times \mathcal{K}$. With the same notations, we have the following isomorphisms

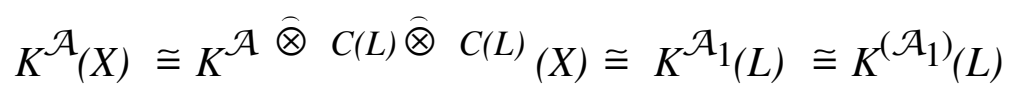

17 More precisely, one has to define an index map parametrized by a Banach bundle, which is also classical. [FM]. 


\section{General equivariant $K$-theory.}

Note : this section is inserted here for the convenience of the reader as an introduction to $\S 6$. It is mainly a summary of results found in [S], [B], [LTX] and [AS].

5.1. Let $A$ be a Banach algebra and $G$ a compact Lie group acting on $A$ via a continuous group homomorphism

$$
G \rightarrow \operatorname{Aut}(A)
$$

where $\operatorname{Aut}(A)$ is provided with the norm topology. We are interested in the category whose objects are finitely generated projective $A$-modules $E$ together with a continuous left action of $G$ on $E$ such that we have the following identity ( $g \in G, a \in A, e \in E$ )

$$
\text { g. }(\text { a.e })=(\text { g.a }) \cdot(\text { g.e })
$$

with an obvious definition of the dots. We define $K_{G}(A)$ as the Grothendieck group of this category $C=\mathcal{P}_{G}(A)$. By the well-known dictionary between bundles and modules, we recover the usual equivariant $K$-theory defined by Atiyah and Segal [S] if $A$ is the ring of continuous maps on a compact space $X$ (thanks to the lemma below). It may also be defined as a suitable semi-direct product of $G$ by $A$ (cf. [B]).

More generally, if $A$ is a $\mathbf{Z} / 2$-graded algebra (where $G$ acts by degree 0 automorphisms), we define the graded equivariant $K$-theory of $A$, denoted by $\operatorname{GrK}_{G}(A)$, as the Grothendieck group of the forgetful functor

$$
C^{0,1} \rightarrow C
$$

where $C^{0,1}$ is the category of "graded objects" in $\mathcal{P}_{G}(A)$ which is defined as $\mathcal{P}_{G}\left(A \otimes C^{0,1}\right)$. In the same spirit as in $\S 1$, we define "derived" groups $K_{G}^{p, q}(A)$ as $G r K_{G}(A \otimes C p, q)$. They satisfy the same formal properties as the usual groups $K_{n}(A)$ (also denoted by $K^{-n}(A)$ with $n=q$ $-p$ ), for instance Bott periodicity. The following key lemma enables us to translate many general theorems of $K$-theory into the equivariant framework :

5.2. LEMMA. Let $E$ be an object of the category $\mathcal{P}_{G}(A)$. Then $E$ is a direct summand of an object of type $A \otimes_{\mathrm{C}} M$ where $M$ is a finite-dimensional $G$-module.

Proof (compare with $[S]$ p. 134). Let us consider the union $\Gamma$ of all finite-dimensional invariant subspaces of the $G$-Banach space $E$. According to a version of the Peter-Weyl theorem theorem quoted in [S] (loc. cit.), this union is dense in $A$. We now consider a set $e_{1}, \ldots, e_{n}$ of generators of $E$ as an $A$-module. Since $\Gamma$ is dense in $A$ and $E$ is projective, one may choose these generators in the subspace $\Gamma$. Let $M_{1}, \ldots, M_{n}$ be finite-dimensional invariant subspaces of $E$ containing $e_{1}, \ldots, e_{n}$ respectively and let $M$ be the following direct sum

$$
M=M_{1} \oplus \ldots \oplus M_{n}
$$

We define an equivariant surjection

$$
\phi: A \otimes_{\mathbf{C}} M \cong\left(A \otimes_{\mathbf{C}} M_{1}\right) \oplus \ldots \oplus\left(A \otimes_{\mathbf{C}} M_{\mathrm{n}}\right) \quad \rightarrow E
$$

between projective left $A$-modules by the formula

$$
\phi\left(\lambda_{1} \otimes m_{1}, \ldots, \lambda_{\mathrm{n}} \otimes m_{n}\right)=\lambda_{1} m_{1}+\ldots+\lambda_{n} m_{n}
$$


This surjection admits a section, which we can average out thanks to a Haar measure in order to make it equivariant. Therefore, $E$ is a direct summand in $A \otimes_{\mathbf{C}} M$ as stated in the lemma.

5.3. As for usual $K$-theory, one may also define equivariant $K$-theory for non unital rings and, using Lemma 5.2 above, prove that any equivariant exact sequence of rings on which $G$ acts

$$
0 \rightarrow A^{\prime} \rightarrow A \rightarrow A^{\prime \prime} \rightarrow 0
$$

induces an exact sequence of equivariant K-groups

$$
K_{G}^{n-1}(A) \rightarrow K_{G}^{n-1}\left(A^{\prime \prime}\right) \rightarrow K_{G}^{n}\left(A^{\prime}\right) \rightarrow K_{G}^{n}(A) \rightarrow K_{G}^{n}\left(A^{\prime \prime}\right)
$$

and an analogous exact sequence in the graded equivariant framework.

5.5. After these generalities, let us assume that $G$ acts on a compact space $X$ and let $\mathcal{A}$ be a bundle of algebras modelled on $\mathcal{K}$. We define the (ungraded) equivariant twisted $K$-group $K_{G}^{(\mathcal{A})}(X)$ as $K_{G}(A)$, where $A$ is the Banach algebra of sections of the bundle $\mathcal{A}$. Similarly, if $\mathcal{A}$ is a bundle of graded algebras modelled on $\mathcal{K}$ x $\mathcal{K}$ or $M_{2}(\mathcal{K})$, we define the (graded) equivariant twisted $K$-group as $\operatorname{GrK}_{G}(\mathrm{~A})$, where $A$ is the graded Banach algebra of sections of $\mathcal{A}$.

As seen in $\S 3$, it is also natural to consider free graded $\mathcal{B}$-modules together with a continuous action of $G$ (compatible with the action on $X$ ) and a family of Fredholm operators $D$ which are self-adjoint of degree one, commuting with the action of $G$. With the same ideas as in $\S 3$, we can show that the Grothendieck group of this category is isomorphic to $K_{G}^{\mathcal{A}}(X)$. This is essentially the definition proposed in [AS].

5.6. An example of such twisted equivariant $K$-theory was given at the beginning of the history of twisted $K$-theory. In [DK] $\S 8$, we defined a "power operation"

$$
\mathrm{P}: K^{\mathcal{A}}(X) \rightarrow K_{S_{n}}^{\mathcal{A}^{\otimes} n}(X)
$$

where $S_{n}$ denotes the symmetric group on $n$ letters acting on $\mathcal{A}{ }^{\widehat{\otimes}}$ (in the spirit of Atiyah's paper on power operations [A1]). According to the general philosophy of Adams and Atiyah, one can deduce from this $n^{\text {th }}$ power operation new "Adams operations" (for $n$ odd):

$$
\Psi^{n}: K^{\alpha}(X) \rightarrow K^{\alpha^{n}}(X) \otimes \Omega_{n}
$$

where $\alpha$ belongs to $\mathbf{Z} / 2 \times H^{1}(X ; \mathbf{Z} / 2) \times H^{3}(X ; \mathbf{Z})$ and $\Omega_{n}$ denotes the free $\mathbf{Z}$-module generated by the $n^{\text {th }}$ roots of unity in $\mathbf{C}$ (the ring of cyclotomic integers). One can prove, following [DK], that these additive maps $\Psi^{n}$ satisfy all the required properties proved by Adams. We shall come back to these operations in $\S 7$, making the link with operations recently defined by Atiyah and Segal [AS2].

5.7. In order to fix ideas, let us consider the graded version of equivariant twisted $K$-theory $K_{G}^{\mathcal{A}}(X)$, where $\mathcal{A}$ is modelled on $\mathbf{C} \times \mathbf{C}$ with the obvious grading. We have again a "Thom isomorphism" : 


$$
t: K_{G}^{\mathcal{A} \hat{\otimes} C(\mathcal{V})}(X) \rightarrow K_{G}^{\mathcal{A}}(V)
$$

whose proof is the same as in the non equivariant case (using elliptic operators). On the other hand, if $L$ is the orientation line bundle of $\mathcal{A}$, the group $G$ acts on $L$ in a way compatible with the action on $X$. Therefore, $\mathcal{A}_{1}=\mathcal{A} \otimes C(L)$ is an algebra bundle with trivial orientation. and $\mathcal{A}_{1} \otimes C^{0,1}$ is naturally isomorphic to $\mathcal{A}_{1} \times \mathcal{A}_{1}=\mathcal{A}_{1}[x] /\left(x^{2}-1\right)$. As in 4.4 , this shows that the graded twisted group $K_{G}^{\mathcal{A}}(X)$ is naturally isomorphic to the ungraded twisted $K$-group $K_{G}^{\left(\mathcal{A}_{1}\right)}(L)$. The same method shows that we can reduce graded twisted $K$-groups to ungraded ones if $\mathcal{A}$ is a bundle of graded algebras modelled on $M_{2}(\mathcal{K})$.

5.8. Let us mention finally one of the main contributions of Atiyah and Segal to the subject ([AS] $\S 6$, see also [CTX]), which we interpret in our language. One is interested in algebra bundles $\mathcal{A}$ on $X$ modelled on $\mathcal{K}$, provided with a left $G$-action. The isomorphism classes of such bundles are in bijective correspondence with principal bundles $P$ over $P U(H)$ (acting on the right) together with a left action of $G$.

Such an algebra bundle $\mathcal{T}$ is called "trivial" if $\mathcal{T}$ may be written as the bundle of algebras of compact operators in $\operatorname{End}(V)$, where $V$ is a $G$-Hilbert bundle. Equivalently, this means that the structure group of $\mathcal{T}$ can be lifted equivariantly to $U(H)$ (in a way compatible with the $G$ action).

We now say that two such algebra bundles $\mathcal{A}$ and $\mathcal{A}$ ' are equivalent if there exist two trivial algebra bundles $\mathcal{T}$ and $\mathcal{T}^{\prime}$, such that $\mathcal{A} \otimes \mathcal{T}$ and $\mathcal{A}^{\prime} \otimes \mathcal{T}^{\prime}$, are isomorphic as $G$-bundles of algebras. The quotient is a group since the dual of $\mathcal{A}$ is its inverse via the tensor product of principal $P U(H)$-bundles. This is the "equivariant Brauer group" $\mathrm{Br}_{G}(X)$.

A closely related definition (in the framework of $C^{*}$-algebras and for a locally compact group $G$ ) has been given in [CKRW]. It is very likely that it coincides with this one for compact Lie groups, in the light of a very interesting filtration described in this paper, which is probably associated to a spectral sequence.

5.9. THEOREM (cf. [AS] prop. 6.3). Let $X_{G}=E G \mathrm{x}_{G} X$ be the Borel space associated to $X$. Then the natural map

$$
B r_{G}(X) \rightarrow B r\left(X_{G}\right)
$$

is an isomorphism.

5.10. The interest of this theorem lies in the fact that the equivariant $K$-theories $K_{G}(\Gamma(X, \mathcal{A}))$ and $K_{G}\left(\Gamma\left(X, \mathcal{A}^{\prime}\right)\right)$ are isomorphic if $\mathcal{A}$ and $\mathcal{A}^{\prime}$ are equivalent. This follows from the wellknown Morita invariance in operator $K$-theory. We shall study concrete applications of this principle in the next section. 


\section{Some computations of twisted equivariant $K$-groups.}

6.1. Let us look at the particular case of the ungraded twisted $K$-groups $K_{G}^{(\mathcal{A})}(X)$ where $G$ is a finite group acting on the trivial bundle of algebras $\mathcal{A}=X \times M_{n}(\mathbf{C})$ via a group homomorphism $G \rightarrow P U(n)^{18}$. We define $\tilde{G}$ as the pull-back diagram

$$
\begin{aligned}
& \tilde{G} \rightarrow S U(n) \\
& \downarrow \quad \downarrow \\
& G \rightarrow P U(n)
\end{aligned}
$$

Therefore, $\tilde{G}$ is a central covering of $G$ with fiber $\mu_{n}$ (whose elements are denoted by Greek letters such as $\lambda$ ). The following definition is already present in [K4] $\$ 2.5($ for $n=2)$ :

6.2. DEFINITION. A finite-dimensional representation $\rho$ of $\tilde{G}$ is of "linear type" if $\rho(\lambda u)=\lambda \rho(u)$ for any $\lambda \in \mu_{n}$.

We now consider the category $E_{\tilde{G}}^{\mathcal{A}}(X)_{l}$ whose objects are $\tilde{G}$ - $\mathcal{A}$-modules as before, except that we request that the $\tilde{G}$-action be of linear type and commute with the action of $\underline{\mathcal{A}}$. By Morita invariance, $E_{\tilde{G}}^{\mathcal{A}}(X)_{l}$ is equivalent to the category $E_{\tilde{G}}(X)_{l}$ of finite-dimensional $\tilde{G}$ bundles on $X$, the action of $\tilde{G}$ on the fibers being of linear type .

6.3. THEOREM ${ }^{19}$. The (ungraded) twisted $K$-theory $K_{G}^{(\mathcal{A})}(X)$ is canonically isomorphic to the Grothendieck group of the category $E_{\tilde{G}}(X)_{l}$.

Proof. One just repeats the argument in the proof of Theorem 2.6 in [K4], where $A$ is a Clifford algebra $C(V)$ and $\mathbf{Z} / 2$ plays the role of $\mu_{n}$. We simply "untwist" the action of $G$ thanks to the formula $(\mathrm{F})$ written explicitly in the proof of 2.6 (loc. cit.).

6.4. For $\mathcal{A}=X \times A$ with $A=M_{n}(\mathbf{C})$, the previous argument shows that $K_{G}^{(\mathcal{A})}(X)$ is a subgroup of the usual equivariant $K$-theory $K_{\tilde{G}}(X)$. From now on, we shall write $K_{G}^{(A)}(X)$ instead of $K_{G}^{(\mathcal{A})}(X)$. Similarly, in the graded case $\left(A=M_{n}(\mathbf{C}) \times M_{n}(\mathbf{C})\right.$ or $\left.M_{2 n}(\mathbf{C})\right)$, we shall write $K_{G}^{A}(X)$ instead of $K_{G}^{\mathcal{A}}(X)$. If $X$ is a point and $G$ is finite, $K_{G}^{(A)}(X)$ is just the $K$-theory of the semi-direct product $G \prec A$.

6.5. THEOREM. Let $G$ be a finite group acting on the algebra of matrices $A=M_{n}(\boldsymbol{C})$ and let $\tilde{G}$ be the central extension $G$ by $\mu_{n}$ described in 6.1. Then, for $X$ reduced to a point, the group $K_{G}^{(\mathcal{A})}(X)=K(G \prec A)$ is a free group of rank the number of conjugacy classes in $G$ which split into $n$ conjugacy classes in $\tilde{G}$.

${ }^{18}$ However, we don't assume that $G$ acts trivially on $X$ in general.

19 There is an obvious generalization when $A$ is infinite-dimensional. However, for our computations, we restrict ourselves to the finite-dimensional case. 
Proof. We can apply the same techniques as the ones detailed in [K4] § 2.6/12 (for $n=2$ ). By the theory of characters on $\tilde{G}$, one is looking for functions $f$ on $\tilde{G}$ (which we call of "linear type") such that

1) $f\left(h g h^{-1}\right)=f(g)$

2) $f(\lambda x)=\lambda f(x)$ if $\lambda$ is an $n^{\text {th }}$ root of the unity

The $\mathbf{C}$-vector space of such functions is in bijective correspondence with the space of functions on the set of conjugacy classes of $G$ which split into $n$ conjugacy classes of $\tilde{G}$.

6.6. Like the Brauer group of a space $X$, one may define in a similar way the Brauer group $\operatorname{Br}(G)$ of a finite group $G$ by considering algebras $A=M_{n}(\mathbf{C})$ as above with a $G$-action (see [FW] for a broader perspective ; this is also a special case of the general theory of Atiyah and Segal mentioned at the end of $\S 5$ ). From the diagram written in 6.1, one deduces a cohomology invariant

$$
w_{2}(A) \in H^{2}\left(G ; \mu_{n}\right)
$$

and therefore (via the Bockstein homomorphism) a second invariant $W_{3}(A) \in H^{2}\left(G ; S^{1}\right)=$ $H^{2}(G ; \mathbf{Q} / \mathbf{Z})=H^{3}(G ; \mathbf{Z})$. It is easy to show that this correspondence induces a well-defined map

$$
W_{3}: \operatorname{Br}(G) \rightarrow H^{3}(G ; \mathbf{Z})
$$

The following theorem is a very special case of 5.9 in a more algebraic situation.

6.7. THEOREM. Let $G$ be a finite group. Then the previous homomorphism

is bijective.

$$
W_{3}: \operatorname{Br}(G) \rightarrow H^{3}(G ; \mathbf{Z})
$$

Proof. First of all, we remark that $H^{3}(G ; \mathbf{Z}) \cong H^{2}(G ; \mathbf{Q} / \mathbf{Z})$ is the direct limit of the groups $H^{2}\left(G ; \mu_{m}\right)$ through the maps $H^{2}\left(G ; \mu_{m}\right) \rightarrow H^{2}\left(G ; \mu_{p}\right)$ when $m$ divides $p$. This stabilization process corresponds on the level of algebras to the tensor product $A \mapsto A \otimes \operatorname{End}(V)$, where $V$ is a $G$-vector space of dimension $p / m$. Therefore, the map $W_{3}$ is injective.

The proof of the surjectivity is deeper (see also 5.9). It relies on a much more general result proved by A. Fröhlich and C.T.C. Wall [FW] about the equivariant Brauer group of an arbitrary field $k$ : there is a split exact sequence (with their notations)

$$
0 \rightarrow \operatorname{Br}(k) \rightarrow B M(k, G) \rightarrow H^{2}(G ; U(k)) \rightarrow 0
$$

where $B r(k)$ is the usual Brauer group of $k, U(k)$ is the group of invertible elements in $k$ and $B M(k, G)$ is a group built out of central simple algebras over $k$ with a $G$-action. Since $\operatorname{Br}(\mathbf{C})=0$ and $H^{2}(G ; U(k))=H^{2}(G ; \mathbf{Q} / \mathbf{Z})$, the theorem is an immediate consequence.

6.8.1. Remark Let us consider an arbitrary central extension $\tilde{G}_{1}$ of $G$ by $\mu_{n} \cong \boldsymbol{Z} / n$ associated to a cohomology class $c \in H^{2}(G ; \mathbf{Z} / n)$. We are interested in the set of elements $g$ of $G$ such that the conjugacy class $<\mathrm{g}>$ splits into $n$ conjugacy classes in $\tilde{G}_{1}$. This set only depends on the image of $c$ in $H^{3}(G ; \mathbf{Z})$ via the Bockstein homomorphism $H^{2}(G ; \mathbf{Z} / n) \rightarrow H^{3}(G ; \mathbf{Z})$ (cf. 6.6). In other words, two central extensions of $G$ by $\mathbf{Z} / \mathbf{n}$ with the same associated image by the Bockstein homomorphism have the same set of $n$-split conjugacy classes. 
In order to show this fact, let us consider the following diagram

$$
\begin{array}{ccc}
\mu_{n} & \rightarrow & \mu_{n m} \\
\downarrow & & \downarrow \\
\tilde{G}_{1} & \rightarrow & \tilde{G} \\
\pi \downarrow & & \downarrow \\
G & = & G
\end{array}
$$

and an element $g_{1}$ of $\tilde{G}_{1}$. The conjugacy class of $g=\pi\left(g_{1}\right)$ splits into $n$ conjugacy classes in $\tilde{G}_{1}$ if and only if there is a trace function $f$ on $\tilde{G}_{1}$ with values in $\mathbf{C}$ such that $f\left(g_{1} c\right)=f\left(g_{1}\right) c$ when $c \in \mu_{n}$. Such a trace function extends obviously to $\tilde{G}$, which yields to the result, since the direct limit of the groups $H^{2}(G ; \mathbf{Z} / n m)$ is precisely $H^{2}(G ; \mathbf{Q} / \mathbf{Z})=H^{3}(G ; \mathbf{Z})$.

6.8.2. The previous remark may be generalized as follows : let $\tilde{G}_{1}$ and $\tilde{G}$ be two group extensions (not necessary central) of $G$ by abelian groups $C_{1}$ and $C$ of orders $m_{1}$ and $m$ respectively, such that the following diagram commutes (with $\alpha$ injective) :

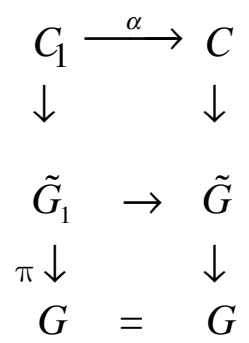

The previous argument shows that if an element $g$ of $G$ splits into $m$ conjugacy classes in $\tilde{G}$, it splits into $m_{1}$ conjugacy classes in $\tilde{G}_{1}$ : take trace functions $f$ on $\tilde{G}$ with values in $C$ such that $f(g c)=f(g)$ c (we write multiplicatively the abelian group $C$ ). The converse is true if the extension of $G$ by $C$ is central.

6.9. Let us now assume that $X$ is not reduced to a point. We can use the Baum-Connes-KuhnSlominska character $[\mathrm{BC}][\mathrm{Kuh}][\mathrm{S}]$ which is defined on $K_{\Gamma}(X)$ (for any finite group $\Gamma$ ), with values in the following direct sum $\underset{<\gamma>}{\oplus} H^{\text {even }}\left(X^{\gamma}\right)^{C(\gamma)}$. In this formula, $<\gamma>$ runs through all the conjugacy classes of $\Gamma, C(\gamma)$ being the centralizer of $\gamma$ (the cohomology is taken with complex coefficients). One of the main features of this "Chern character"

$$
K_{\Gamma}(X) \rightarrow \underset{<\gamma>}{\oplus} H^{e v e n}\left(X^{\gamma}\right) C(\gamma)
$$

is the isomorphism it induces between $K_{\Gamma}(X) \otimes_{Z} \mathbf{C}$ and the cohomology with complex coefficients on the right-hand side. If $E$ is a $\Gamma$-vector bundle, the map is defined explicitly by Formula 1.13 p. 170 in [BC]. 
Let us now take for $\Gamma$ the group $\tilde{G}$ previously considered and let us analyse the formula in this case. We shall view the right-hand side not just as a function on the set of conjugacy classes $\langle\gamma\rangle$, but as a function $f$ on the full group $\Gamma$ with certain extra properties which we are going to explain.

If we replace $\gamma$ by $\gamma^{\prime}$ such that $\gamma^{\prime}=\sigma \gamma \sigma^{-1}$, we have a canonical isomorphism $\sigma^{*}$ :

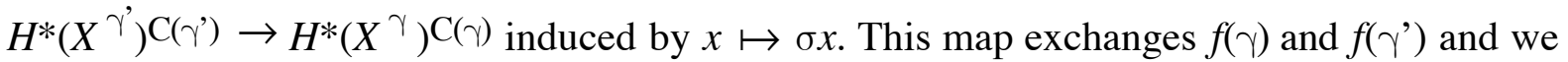
have the relation $f(\gamma)=\sigma^{*}\left(f\left(\gamma^{\prime}\right)\right)$, which says that $f$ is essentially a function on the conjugacy classes.

On the other hand, if the action of $\tilde{G}$ is of linear type, we have an extra relation, an easy consequence of the formula in [BC], which is $f(\mu \gamma)=\mu f(\gamma)$ when $\mu$ is an $n^{\text {th }}$ root of unity. To summarize, we get the following theorem.

6.10. THEOREM. Let $G$ be a finite group and $A=M_{n}(\mathbf{C})$ with a $G$-action. Then the ungraded twisted equivariant $K$-theory $K_{G}^{(A)}(X)$ is a subgroup of the equivariant $K$-theory $K_{\widehat{G}}(X)$, where $\tilde{G}$ is the pull-back diagram

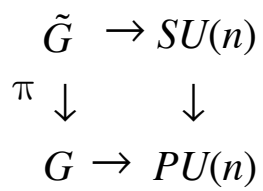

More precisely, $K_{G}^{(A)}(X) \otimes_{\mathbf{Z}} \mathbf{C}$ may be identified with the $\mathbf{C}$-vector space of functions $f$ on $\Gamma=\tilde{G}$ with $f(\gamma)$ in $H^{\text {even }}\left(X^{g}\right) C(\gamma), \pi(\gamma)=g$, such that the following two identities hold:

1) If $\gamma^{\prime}=\sigma \gamma \sigma^{-1}$, one has $\mathrm{f}(\gamma)=\sigma^{*}\left(\mathrm{f}\left(\gamma^{\prime}\right)\right)$, according to the formula above

2) $\mathrm{f}(\mu \gamma)=\mu \mathrm{f}(\gamma)$ if $\mu$ is an $n^{\text {th }}$ root of unity

In particular, if $X$ is reduced to a point, we have $\sigma^{*}=I d$ and $K_{G}^{(A)}(X)$ is free with rank the number of conjugacy classes of $G$ which split into $n$ conjugacy classes in $\tilde{G}$, as we have seen in 6.5 .

6.11. Remarks. This theorem is not really new. In a closely related context, one finds similar results in $[\mathrm{AR}]$ and $[\mathrm{TX}]$. We should also notice that the same ideas have been used before in [K4] for representations of "linear type". Finally, the theorem easily extends to locally compact spaces if we consider cohomology with compact supports on the right-hand side.

6.12. THEOREM. Let A be any finite-dimensional graded semi-simple complex algebra with a graded action of a finite group $G$. Then the graded $K$-theory $G_{0} K_{0}\left(A^{\prime}\right) \oplus G r K_{1}\left(A^{\prime}\right)$ of the semi-direct product $A^{\prime}=G \prec A$ is a non trivial free $\mathbf{Z}$-module. In particular, if $V$ is a real finite-dimensional vector space with a $G$ action, the group $K_{G}^{A}(V) \oplus K_{G}^{A}(V \oplus 1)$ is free non trivial thanks to the Thom isomorphism.

Proof. The algebra $G \prec A$ is graded semi-simple over the complex numbers. Therefore, it is a direct sum of graded algebras Morita equivalent to $M_{n}(\mathbf{C})$ x $M_{n}(\mathbf{C})$ or $M_{2 n}(\mathbf{C})$. In both cases, the graded $K$-theory is non trivial. The last part of the theorem follows from 4.2. 
The following theorem is a direct consequence of the previous considerations :

6.13. THEOREM. Let us now assume that $A=M_{2 n}(\mathbf{C})$ is $G$-oriented as a graded algebra :in other words, there is an involutive element $\varepsilon$ of $A$ of degree 0 which commutes with the action of $G$ and commutes (resp. anticommutes) with the elements of A of degree 0 (resp. 1). Then, the graded $K$-theory $G r K *\left(A^{\prime}\right)$, with $A^{\prime}=G \prec A$, is a finitely generated free module concentrated in degree 0. More precisely, one has $G r K_{0}\left(A^{\prime}\right)=K\left(A^{\prime}\right)$ and $G r K_{1}\left(A^{\prime}\right)=0$. In particular, if $V$ is an even-dimensional real vector space and if $A \otimes C(V)$ is $G$-oriented, we have (via the Thom isomorphism)

$$
K_{G}^{A}(V)=K_{G}^{A \widehat{\otimes} C(V)}(P)=K(G \prec(A \widehat{\otimes} C(V))) \text { and } K_{G}^{A}(V \oplus 1)=0
$$

where $P$ is a point. If we write $A \otimes C(V)$ as an algebra of matrices $M_{r}(\mathbf{C})$ with a representation $\rho$ of $G$ and call $\tilde{G}$ the associated central extension by $\mu_{r^{\prime}}$ the rank of $K_{G}^{A}(V)$ is the number of conjugacy classes of $G$ which split into $r$ conjugacy classes in $\tilde{G}$.

In the abelian case, the following two theorems are related to results obtained by $\mathrm{P}$. Hu and I. Kriz [HK], using different methods.

6.14. THEOREM. Let us consider the algebra $A=M_{n}(\mathbf{C})$ provided with an action of an abelian group $G$. Then the ungraded twisted $K$-theory $K_{G}^{(A)}(P)_{*}=K_{*}\left(A^{\prime}\right)$, with $A^{\prime}=G \prec A$, is concentrated in degree 0 and is a free $\mathbf{Z}$-module. If we tensor this group with the rationals and if we look at it as an $R(G) \otimes \mathbf{Q}=\mathbf{Q}[G]$-module, it may be identified with $R\left(G^{\prime}\right) \otimes \mathbf{Q}$ for a suitable subgroup $G^{\prime}$ of $G$. In particular, the rank of $K_{0}\left(A^{\prime}\right)$ divides the order of $G$.

Proof. The first part of the theorem is a consequence of the previous more general considerations. As we have shown before, the algebra $A$ ' gives rise to the following commutative diagram

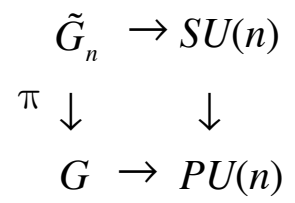

the fibers of the vertical maps being $\mu_{n}$. The subset of elements $\tilde{g}$ in $\tilde{G}_{n}$ such that $\pi(\tilde{g})$ splits into $n$ conjugacy classes is just the center $Z\left(\tilde{G}_{n}\right)$ of $\tilde{G}_{n}$ (since $G$ is abelian). Let us put $\Gamma_{n}=$ $\pi\left(Z\left(\tilde{G}_{n}\right)\right)$. Then $K\left(A^{\prime}\right)$ may be written as $K_{G}^{A}(P)$ where $P$ is a point. According to Theorem 6.10 , this is the subgroup of the representation ring of $\tilde{G}_{n}$ generated by representations of linear type. At this stage, it is convenient to make $n=\infty$ by extension of the roots of unity, so that we have an extension of $G$ by $\mathbf{Q} / \mathbf{Z}$

$$
\mathbf{Q} / \mathbf{Z} \rightarrow \tilde{G} \rightarrow G
$$

(the "linear type" finite-dimensional representations of $\tilde{G}$ are the same as the original linear 
type finite-dimensional representations of $\left.\tilde{G}_{n}\right)$. We call $R(\tilde{G})_{1}$ the associated Grothendieck group. By the theory of characters, we see that $R(\tilde{G})_{1} \otimes \mathbf{Q}$ is isomorphic to $R(Z(\tilde{G}))_{1} \otimes \mathbf{Q}$, since the characters of such linear type representations of $\tilde{G}$ vanish outside $Z(\tilde{G})$. On the other hand, if we denote by $G^{\prime}$ the image of $Z(\tilde{G})$ in $G$, the extension of abelian groups

$$
\mathbf{Q} / \mathbf{Z} \rightarrow \mathrm{Z}(\tilde{G}) \rightarrow \mathrm{G}^{\prime}
$$

splits (non canonically). This means that we can identify $R(\tilde{G})_{1} \otimes \mathbf{Q}$ with the representation ring $R\left(G^{\prime}\right) \otimes \mathbf{Q}$ as an $R(G) \otimes \mathbf{Q}$-module. This proves the last part of the theorem.

6.15. THEOREM. Let us consider a graded algebra $A=M_{2 n}(\mathbf{C})$ provided with a non oriented action of an abelian group $G$ (with respect to the grading). Then the graded twisted $K$-theory $K_{G}^{A}(P)_{*}=\operatorname{Gr} K_{*}\left(A^{\prime}\right)$, with $A^{\prime}=G \prec A$, is concentrated in a single degree ( 0 or 1$)$ and is a free $\mathbf{Z}$-module. If we tensor this group with the complex numbers and if we look at it as an $R(G) \otimes \mathbf{C}=\mathbf{C}[G]$-module, it may be identified with $R\left(G^{\prime}\right) \otimes \mathbf{Q}$ for a suitable subgroup $G$ ' of $G$. In particular, the rank of $K_{G}^{A}(P)_{*}$ divides the order of $G$.

Proof. Let $L$ be the orientation bundle of $A$ (with respect to the action of $G$ ). If we change $A$ into $A \widehat{\otimes} C(L)$ and if we apply the Thom isomorphism theorem, we have to compute $K_{G}^{A}(L)_{*}$ where $G$ acts on $A$ (resp. $L$ ) in an oriented way (resp. non oriented way).

Let us apply Theorem 6.10 in this situation : since $G$ is abelian, the function $f$ of the theorem must be equal to 0 on the elements of $\tilde{G}$ which are not in $Z(\tilde{G})$. Therefore, the relevant group $K_{G}^{A}(L)_{*}$ is reduced to $K_{G},(L)$ (after tensoring with $\mathbf{C}$ and where $G^{\prime}$ is the image of $Z(\tilde{G})$ in $G)$. We now consider two cases :

1) the action $\rho$ of $G^{\prime}$ on $L$ is oriented, in which case we only find $R\left(G^{\prime}\right)$ (with a shift of dimension). Therefore, the dimension of $K_{G}^{A}(L)_{*} \otimes \mathbf{C}$ is the order of $G^{\prime}$ which divides the order of $G$.

2) the action $\rho$ of $G^{\prime}$ on $L$ is not oriented. We then find a direct sum of copies of $\mathbf{C}$, each one corresponding to an element of $G^{\prime}$ such that $\rho\left(g^{\prime}\right)=-1$. The dimension of $K_{G}^{A}(L)_{*} \otimes \mathbf{C}$ is therefore half the order of $G^{\prime}$, hence divides $|G| / 2$.

6.16. Remarks. For an oriented action of $G$ on $M_{2 n}(\boldsymbol{C})$, Theorem 3.5 enables us to reduce the analogous problem in Theorem 6.15 to ungraded twisted $K$-theory, which is done in 6.14 .

On the other hand, as we already mentioned, the two last theorems (for $G$ abelian) are related to results found by P. Hu and I. Kriz [HK].

In [K4] we also perform other types of computations when $A$ is a Clifford algebra and $G$ is any finite group, again using the Thom isomorphism. For instance, if $G$ is the symmetric group $S_{n}$ acting on the Clifford algebra of $\mathbf{R}^{n}$ via the canonical representation of $S_{n}$ on $\mathbf{R}^{n}$, there is a nice relation with the pentagonal identity of Euler (cf. [K4] p. 532).

As a concluding remark, we would like to point out also that the theory $K_{ \pm}(X)$ introduced recently by Atiyah and Hopkins [AHo] is a particular case of twisted equivariant $K$-theory. As 
a matter of fact, it was explicitly present in [K1] $\S 3,40$ years ago, before the formal introduction of twisted $K$-theory !

According to [AHo], the definition of $K_{ \pm}(X)$ (in the complex or real case) is the group

$K_{\mathbf{Z} / 2}\left(X \times \mathbf{R}^{8}\right)$, where $\mathbf{Z} / 2$ acts on $X$ and also on $\mathbf{R}^{8}=\mathbf{R} \times \mathbf{R}^{7}$ by $(\lambda, \mu) \mapsto(-\lambda, \mu)$. According to the Thom isomorphism in equivariant $K$-theory (proved in [K3]), it coincides with an explicit graded twisted $K$-group $K_{Z / 2}^{A}(X)$, as defined in [K1]. Here $A$ is the Clifford algebra $C\left(\mathbf{R}^{2}\right)=$ $C^{1,1}$ of $\mathbf{R}^{2}$ provided with the quadratic form $x^{2}-y^{2}$ and where $\mathbf{Z} / 2$ acts via the involution $(\lambda, \mu) \mapsto(-\lambda, \mu)$ on $\mathbf{R} \times \mathbf{R}$ (this is also mentioned briefly in [AHo] p. 2, footnote 1). This identification is valid as well in the real framework, where we have 8-periodicity.

These groups $K_{Z / 2}^{A}(X)$ were precisely those considered in [K1] $\S 3.3$ in a broader context : $A$ may be any Clifford algebra bundle $C(V)$ (where $V$ is a real vector bundle provided with a non degenerate quadratic form) and $\mathbf{Z} / 2$ may be replaced by any compact Lie group acting in a coherent way on $X$ and $V$. Note that the real and complex Fredholm descriptions for the non twisted case were also considered with great detail independently in [ASi] and [K5]. These descriptions overlap with the results of our $\S 3$ and 4. The paper [K4] gives a method to compute these equivariant $K$-groups, at least rationally, in a more general context, using the equivariant Thom isomorphism.

\section{Operations on twisted $K$-groups.}

Note : this section is a partial synthesis of [DK] (1970) and [AS2] (2005).

7.1. Let us start with the simple case of bundles of (ungraded) infinite $C^{*}$-algebras modelled on $\mathcal{K}$, as in [AS2]. As it was shown in [A1] and [DK], we have a $n^{\text {th }}$ power map

$$
\mathrm{P}: K^{(\mathcal{A})}(X) \rightarrow K_{S_{n}}^{\left(\mathcal{A}^{\otimes n}\right)}(X)
$$

where the symmetric group $S_{n}$ acts on $\mathcal{A}^{\otimes n}$ by permutation of the factors.

7.2. LEMMA. The group $K_{S_{n}}^{\left(\mathcal{A}^{\otimes n}\right)}(X)$ is isomorphic to the group $K_{S_{n}}^{\left(\mathcal{A}^{\otimes n}\right)_{0}}(X)$ where the symbol o means that $S_{n}$ is acting trivially on $\mathcal{A}^{\otimes n}$.

Proof. As we have shown many times in $\S 6$, this "untwisting" of the action of the symmetric group on $\mathcal{A}^{\otimes n}$ is due to the following fact : the standard representation

$$
S_{n} \rightarrow P U\left(H^{\otimes n}\right)
$$

can be lifted into a representation $\rho: S_{n} \rightarrow U\left(H^{\otimes n}\right)$ in a way compatible with the diagonal action of elements of $P U(H)$, a fact which is obvious to check. If $E$ is a module ${ }^{20}$ over $\mathcal{A}^{\otimes n}$ and $S_{n}$, we then have to untwist the action of $S_{n}$ by a new action $*$ defined by

\footnotetext{
$\overline{20}$ As a matter of fact, we should add a unit fibrewise to $\mathcal{A}$ in order to make sense of the module structure.
} 


$$
\mathrm{g} * \mathrm{e}=\rho(\mathrm{g})^{-1}(\mathrm{~g} \cdot \mathrm{m})
$$

This $*$ action of $S_{n}$ now commutes with the action of $\mathcal{A}^{\otimes n}$ (see again and for instance [K4] 2.6).

7.3. Remark. If we take a bundle of finite dimensional algebras modelled on $A=\operatorname{End}(E)$ where $E=\mathbf{C}^{r}$, there is another way to check (functorially) the untwisting : we identify $A^{\otimes n}$ with $\operatorname{End}\left(E^{\otimes n}\right)$ and $\left(A^{\otimes n}\right)^{*}$ with $\operatorname{Aut}\left(E^{\otimes n}\right)$. We have the following commutative diagram

$$
\begin{array}{cc} 
& \operatorname{End}\left(E^{\otimes n}\right) *=\operatorname{Aut}\left(E^{\otimes n}\right) \\
\theta & \downarrow \pi \\
S_{n} \rightarrow \quad & \\
& \operatorname{End}\left(E^{\otimes n}\right)=\operatorname{End}(E)^{\otimes n}
\end{array}
$$

The vertical map sends the invertible element $\alpha$ to the automorphism $\left(u \mapsto \alpha u \alpha^{-1}\right)$. If $\sigma$ is a permutation, the horizontal map sends $\sigma$ to the automorphism

$$
u_{1} \otimes \cdots \otimes u_{n} \mapsto u_{\sigma(1)} \otimes \cdots \otimes u_{\sigma(n)}
$$

while the map $\theta$ sends $\sigma$ to the automorphism of $E^{\otimes n}$ defined by

$$
x_{1} \otimes \cdots \otimes x_{n} \mapsto x_{\sigma(1)} \otimes \cdots \otimes x_{\sigma(n)}
$$

Finally, the composition $\pi \theta$, computed on a decomposable vector of $E^{\otimes n}$ gives the required result :

$$
\begin{aligned}
& \mathrm{x}_{1} \otimes \ldots \otimes \mathrm{x}_{n} \stackrel{\sigma}{\longrightarrow} \mathrm{x}_{\sigma(1)} \otimes \ldots \otimes \mathrm{x}_{\sigma(n)} \stackrel{u}{\longrightarrow} \mathrm{u}_{1}\left(\mathrm{x}_{\sigma(1)}\right) \otimes \ldots \otimes \mathrm{u}_{\mathrm{n}}\left(\mathrm{x}_{\sigma(n)}\right) \\
& \stackrel{\sigma^{-1}}{\longrightarrow} \mathrm{u}_{\sigma(1)}\left(\mathrm{x}_{1}\right) \otimes \ldots \otimes \mathrm{u}_{\sigma(n)}\left(\mathrm{x}_{n}\right)
\end{aligned}
$$

7.4. As it was shown in [A1], a Z-module map

$$
R\left(S_{n}\right) \rightarrow \mathbf{Z}
$$

defines an operation in twisted K-theory by taking the composite of the following maps

$$
K^{(\mathcal{A})}(X) \rightarrow K_{S_{n}}^{\left(\mathcal{A}^{\otimes n}\right)}(X) \cong K_{S_{n}}^{\left(\mathcal{A}^{\otimes n}\right)_{0}}(X) \cong K^{(\mathcal{A})^{\otimes n}}(X) \otimes R\left(S_{n}\right) \rightarrow K^{(\mathcal{A})^{\otimes n}}(X)
$$

This is essentially ${ }^{21}$ what was done in [AS2] $\S 10$, in order to define the $\lambda^{n}$ operation of Grothendieck in this context for instance.

Let us call $F$ (for short) the image of $(E, D)$ by the composite of the maps

${ }^{21}$ The second homomorphism was not explicitly given however. 


$$
K^{(\mathcal{A})}(X) \rightarrow K_{S_{n}}^{\left(\mathcal{A}^{\otimes n}\right)}(X) \rightarrow K_{S_{n}}^{\left(\mathcal{A}^{\otimes n}\right)_{0}}(X) .
$$

We can also define the Adams operations $\Psi^{n}$ with the method described in [DK] (which we intend to generalize later on). For this, we restrict the action of $S_{n}$ to the cyclic group $\mathbf{Z} / n$ identified with the group of $n^{\text {th }}$ roots of the unity. Let us now call $F_{r}$ the subgroup of $F$ where the action of $\mathbf{Z} / n$ is given by $\omega^{r}, \omega$ being a fixed primitive root of the unity. Then $\Psi^{n}(E, D)$ is defined by the following sum

$$
\Psi^{n}(E, D)=\sum_{0}^{n-1} F_{r} \omega^{r}
$$

It belongs formally to $K^{(\mathcal{A})^{\otimes n}}(X) \otimes \Omega_{n}$ where $\Omega_{n}$ is the ring of $n$-cyclotomic integers. However, if $n$ is prime, using the action of the symmetric group $S_{n}$, it is easy to see that $F_{r}$ is isomorphic to $F_{1}$ if $r \neq 1$. Therefore we end up in $K^{(\mathcal{A})^{\otimes n}}(X)$, considered as the subgroup of $K^{(\mathcal{A})^{\otimes n}}(X) \otimes \Omega_{n}$, as it was expected. It is proved in [A1] that this definition of $\Psi^{n}$ agrees with the classical one.

There is another operation in twisted K-theory which is "complex conjugation", denoted classically by $\Psi^{-1}$, which maps $K^{(\mathcal{A})}(X)$ to $K^{(\mathcal{A})^{-1}}(X)$ (if we write multiplicatively the group law in $\operatorname{Br}(X))$. It is shown in [AS2] $\S 10$ how we can combine this operation with the previous ones in order to get "internal" operations in twisted K-theory, i.e. mapping $K^{(\mathcal{A})}(X)$ to itself.

7.5. It is more tricky to define operations in graded twisted $\mathrm{K}$-theory. If $\Lambda$ is a $\mathrm{Z} / 2$ graded algebra, it is no longer true in general that a graded involution of $\Lambda$ is induced by an inner automorphism with an element of degree 0 and of order 2 . A typical example is the Clifford algebra

$$
(\mathbf{C} \oplus \mathbf{C})^{\widehat{\otimes} 2}=\left(C^{0,1}\right)^{\widehat{\otimes} 2}
$$

which may be identified with the graded algebra $M_{2}(\mathbf{C})$. If we put

$$
e_{1}=\left(\begin{array}{cc}
0 & 1 \\
1 & 0
\end{array}\right) \text { and } e_{2}=\left(\begin{array}{cc}
0 & -i \\
i & 0
\end{array}\right)
$$

we see that there is no inner automorphism by an element of order 2 and degree 0 permuting $e_{1}$ and $e_{2}$.

On the other hand, we know by the general theory that the standard representation

$$
\rho: S_{n} \rightarrow \operatorname{Aut}(\mathcal{A})^{\otimes n}
$$

where $\mathcal{A}$ is a bundle of graded algebras modelled on $\mathcal{K}$ x $\mathcal{K}$ or $M_{2}(\mathcal{K})$ can be lifted by inner automorphisms ${ }^{22}$ with elements of degree 0 or 1 . Therefore, if we restrict the representation $\rho$ to the alternating group $A_{n}$, we get indeed a representation from $A_{n}$ to the 0 -degree part of 
$\left(\mathcal{A}^{\widehat{\otimes} n}\right) *$ such that the following diagram commutes

$$
\begin{gathered}
\left(\mathcal{A}^{\widehat{\otimes} n}\right)^{*} \\
\downarrow \\
A_{n} \rightarrow \operatorname{Aut}(\mathcal{A})^{\otimes n}
\end{gathered}
$$

7.6. In order to define the operations $\Psi^{n}$ in graded twisted K-theory, we now follow the scheme developed in [DK] and choose the description of twisted K-theory given by a family of Fredholm operators (cf. 3.14 ). The power map

$$
K^{\mathcal{A}}(X) \rightarrow K_{S_{n}}^{\mathcal{A}^{\otimes} n}(X)
$$

still makes sense : we just take the $n^{\text {th }}$ (graded) tensor product of $(E, D)$ by itself with the notations of $3.12 / 14$. The graded representation of $S_{n}$ in $\mathcal{B}^{\otimes}$ does not lift in general to a representation of $S_{n}$ in the 0 -degree part of $\left(\mathcal{B}^{\widehat{\otimes} n}\right)^{*}$. However, as we have seen before, it is the case if we restrict this representation to the alternating group $A_{n}$. Therefore, any $\mathbf{Z}$-module map $R\left(A_{n}\right) \rightarrow \mathbf{Z}$ gives rise to an operation in graded twisted $\mathrm{K}$-theory which is the following composite :

$$
K^{\mathcal{A}}(X) \rightarrow K_{A_{n}}^{\mathcal{A}^{\Phi} n}(X) \rightarrow K^{\mathcal{A}^{\bar{\otimes} n}}(X) \otimes R\left(A_{n}\right) \rightarrow K^{\mathcal{A}^{\bar{\otimes} n}}(X)
$$

Among these operations, we should mention the Adams operations $\Psi^{n}$ as defined in [DK]. For this we restrict even more the action of $A_{n}$ to the cyclic group $\mathbf{Z} / n$ with $n$ odd (such that $\mathbf{Z} / n$ is a subgroup of $A_{n}$ ).

The Adams operation $\Psi^{n}$ is then given by the same formula as in 7.4

$$
\Psi^{n}: K^{\mathcal{A}}(X) \rightarrow K^{\mathcal{A}^{\otimes n}}(X) \otimes \Omega_{n}
$$

where $\Omega_{n}$ is the ring of $n$-cyclotomic integers. We can copy the proof in [DK] to show that this operation is additive and multiplicative up to canonical isomorphisms (see Theorem 30, p. 23 in $[\mathrm{DK}])$.

7.7. Although, we have been forced to introduce the alternating group in order to define operations in graded twisted K-theory (see 7.9 below), there are important cases where we can stay in the classical situation described at the beginning of the section. In particular, if $\mathcal{A}$ is a bundle of oriented graded algebras modelled on $M_{2}(\mathcal{K})$, we have seen in 3.5 that the two groups $\mathrm{K}^{\mathcal{A}}(\mathrm{X})$ and $\mathrm{K}(\mathcal{A})(\mathrm{X})$ coincide. As a matter of fact, in this case, we have a commutative diagram

\footnotetext{
${ }^{22}$ Again, we must add a unit in order to make sense of $\left(\mathcal{A}^{\otimes}\right)^{*}$. Another possibility which we will consider in 7.6 is to work with the bundle $\mathcal{B}$ rather than $\mathcal{A}$ (see 2.6).
} 


$$
\begin{gathered}
\downarrow \\
S_{n} \rightarrow \mathrm{PU}(\mathrm{H} \oplus \mathrm{H})
\end{gathered}
$$

with $S_{n}$ instead of $A_{n}$ since the image of $S_{n}$ by the horizontal map is inside $\mathrm{PU}^{0}(\mathrm{H} \oplus \mathrm{H})$, with the notation of 3.4 .

7.8. As a concluding remark, we should notice that the image of $\Psi^{n}$ as defined in 7.6 is not arbitrary. If $k$ and $n$ are coprimes, the multiplication by $k$ on the group $\mathbf{Z} / n$ defines an element of the symmetric group $S_{n}$. The signature of this permutation is called the Legendre symbol $\left(\begin{array}{l}k \\ n\end{array}\right)$. We denote by $F_{r}$ (as in 7.4) the element associated to the eigenvalue $\mathrm{e}^{2 \mathrm{i} \pi r}$. Then we see that $F_{r}$ and $F_{r k}$ are isomorphic if the Legendre symbol $\left(\begin{array}{l}k \\ n\end{array}\right)$ is equal to 1 (consider again the multiplication by $k$ mod. $n$ ). If $n$ is prime for instance, $\Psi^{n}(E)$ may therefore be written in the following way :

$$
\Psi^{n}(E)=F_{0}+\sum_{\left(\begin{array}{l}
k \\
n
\end{array}\right)=1} U \omega^{k}+\sum_{\left.l_{n}^{k}\right)=-1} V \omega^{k}
$$

where $U$ (resp. $V$ ) is any $F_{k}$ with Legendre's symbol equal to 1 (resp. -1).

We should also notice, following [AS2], that there is no problem to define the Adams operation $\Psi^{-1}$ in graded twisted K-theory and combine it with compositions of the $\Psi^{n}$ 's in order to define "internal" operations.

7.9. The simplest non-trivial example of such an operation is

$$
\Psi^{n}: \mathbf{Z} \cong K^{1}\left(S^{1}\right) \rightarrow K^{n}\left(S^{1}\right) \otimes \Omega_{n}
$$

where $n$ is a product of different odd primes. Since the operation $\Psi^{n}$ on $K^{2}\left(S^{2}\right)$ is the multiplication by $n$, we deduce that $\theta=\sqrt{(-1)^{(n-1) / 2} n}$ belongs to $\Omega_{n}$ (a well-known result) and that $\Psi^{n}$ on $K^{1}\left(S^{1}\right)$ is essentially the inclusion of $\mathbf{Z}$ in $\Omega_{n}$ defined by $1 \mapsto \theta$. 


\section{REFERENCES}

[AR] A. ADEM and Y. RUAN. Twisted Orbifold $K$-theory. Communications in Mathematical Physics 237, pp. 533--556 (2003).

[A1] M. F. ATIYAH. $K$-theory. Notes by D.W. Anderson. Second edition. Advanced Book Classics. Addison-Wesley Publishing Company (1989).

[A2] M. F. ATIYAH. Bott periodicity and the index of elliptic operators. The Quarterly Journal of Math. 1968, pp. 113-140 (1968).

[AH] M. F. ATIYAH and F. HIRZEBRUCH. Riemann-Roch theorems for differentiable manifolds. Bull. Amer. Math Society, p. 276-281 (1959).

[AHo] M. F. ATIYAH and M. HOPKINS. A variant of K-theory : $K_{ \pm}$. Topology, geometry and quantum field theory. London Math. Soc. Lecture Note Ser., 308, Cambridge Univ. Press, Cambridge, pp. 5-17 (2004).

[AS] M.F. ATIYAH and G. SEGAL. Twisted $K$-theory. Ukr. Mat. Visn. 1, pp. 287-330 (19) (2004) MR 2172633, ArXiv math.KT/0407054 (2005).

[AS2] M.F. ATIYAH and G. SEGAL. Twisted $K$-theory and cohomology. ArXiv math. KT/0510674 (2005).

[ASi] M.F. ATIYAH and I.M. SINGER. Index theory for skew adjoint Fredholm operators. Publ. Math. IHES Paris 37, pp. 5-26 (1969). [Compare with [K5] ]

[BC] P. BAUM and A. CONNES. Chern character for discrete groups in "A fête of Topology", pp. 163-232. Academic Press, Boston, MA (1988).

[B] B. BLACKADAR. $K$-theory for operator algebras. Second edition. Cambridge University Press (1998).

[BCMMS] P. BOUWKNEGT, A.L. CAREY, V. MATHAI, M.K. MURRAY and D. STEVENSON. Twisted $K$-theory and $K$-theory of Bundle Gerbes. Comm. Math. Phys. 228, pp. 17-45 (2002).

[Ca] A.L. CAREY and Bai-Ling WANG. Thom isomorphism and push-forward maps in twisted $K$-theory. math. KT/0507414 (2006).

[CKRW] D. CROCKER, A. KUMJIAN, I. RAEBURN, D.P. WILLIAMS. An equivariant Brauer group and action of groups on C*-algebras. J. Funct. Anal. 146, pp. 151-184 (1997).

[DD]. J. DIXMIER et A. DOUADY. Champs continus d'espaces hilbertiens et de C*algèbres. Bull. Soc. Math. France 91, pp. 227-284 (1963).

[DK] P. DONOVAN and M. KAROUBI. Graded Brauer groups and $K$-theory with local coefficients. Publ. Math. IHES 38, p. 5-25 (1970). French summary in : Groupe de Brauer et coefficients locaux en $K$-théorie. Comptes Rendus Acad. Sci. Paris, t. 269, pp. 387-389 (1969). 
[FM] A.T. FOMENKO and A.S. MISCENKO. The index of elliptic operators over C*algebras. Izv. Akad. Nauk. SSSR, Ser. Mat. 43, pp. 831-859 (1979).

[FHT] D.S. FREED, M. HOPKINS and C. TELEMAN. Twisted $K$-theory and loop group representations. ArXiv math/0206237, 0312155 and 0511232.

[FW] A. FROHLICH and C.T.C. WALL. Equivariant Brauer groups. Contemp. Math. 272, pp. 57-71 (2000).

[G] A. GROTHENDIECK. Le groupe de Brauer. Séminaire Bourbaki 290 (1965).

[J] K. JANICH. Vektorraumbündel und der Raum der Fredholm-operatoren. Math. Ann. 161, pp. 129-142 (1965).

[K1] M. KAROUBI. Algèbres de Clifford et K-théorie. Ann. Sci. Ecole Norm. Sup. (4), pp. 161-270 (1968).

[K2] M. KAROUBI. Algèbres de Clifford et opérateurs de Fredholm. Springer Lecture Notes in Maths $N^{\circ} 136$, p. 66-106 (1970). Summary in Comptes Rendus Acad. Sci. Paris, t. 267, pp. 305 (1968).

[K3] M. KAROUBI. Sur la $K$-théorie équivariante. Springer Lecture Notes in Math. № 136 , pp. 187-253 (1970).

[K4] M. KAROUBI. Equivariant $K$-theory of real vector spaces and real projective spaces. Topology and its applications 122, pp. 531-546 (2002).

[K5] M. KAROUBI. Espaces classifiants en $K$-théorie. Trans. of the Amer. Math. Soc., Vol 147, pp. 75-115 (1970). [compare with [ASi]].

[KH] I. KRIZ and P. HU. The $R O(G)$-graded coefficients of $(\mathbf{Z} / 2)^{n}$-equivariant $K$-theory. ArXiv math. KT/0609067 (2006).

[Kuh] N. KUHN. Character rings in algebraic topology, Advances in Homotopy Theory, Proceedings of Cortona 1988, LMS Lecture Note Series 139 pp. 111-126(1989).

[Ku] N. KUIPER. The homotopy type of the unitary group in Hilbert space. Topology 3, pp. 19-30 (1965).

[LTX] C. LAURENT, J.-L. TU and P. XU. Twisted K-theory of differential stacks. Ann. Scient. Ec. Norm. Sup. 37, pp. 841-910 (2004).

[MS] V. MATHAI and D. STEVENSON. On a generalized Connes-Hochschild-KostantRosenberg theorem. Advances in Math. 200, pp. 303-335 (2006).

[Mi] E.A. MICHAEL. Convex structures and continuous selections. Canad. J. Math., vol. 11, pp. 556-575 (1959).

[R] J. ROSENBERG. Continuous-trace algebras from the bundle theoretic point of view. J. Austral. Math. Soc. A 47, pp. 368-381 (1989).

[S] G. SEGAL. Equivariant $K$-theory. Publ. Math. de l'IHES, tome 34, pp. 129-151 (1968). 
[S] J. SLOMINSKA. On the equivariant Chern homomorphism. Bulletin de l'Académie Polonaise des Sciences, vol. XXIV, N 10, pp. 909-913 (1976).

[TX] J.-L. TU and Ping XU. The Chern character for twisted $K$-theory of orbifolds. ArXiv math. KT/0505267 (2005).

[Wi] E. WITTEN. D-branes and K-theory. J. High Energy Phys. 12, paper 19, hepth/0007175 (1998).

[W] R. WOOD. Banach algebras and Bott periodicity. Topology, vol. 4, pp. 371-389 (1966). 